OPEN ACCESS

Edited by:

Stephanie DeWitte-Orr, Wilfrid Laurier University, Canada

Reviewed by: Shawna L. Semple, Wilfrid Laurier University, Canada Jondavid de Jong, Glysantis, Canada

*Correspondence: Shun Li

bob@ihb.ac.cn Yong-An Zhang yonganzhang@mail.hzau.edu.cn

${ }^{\dagger}$ These authors have contributed equally to this work

Specialty section: This article was submitted to Comparative Immunology, a section of the journal

Frontiers in Immunology

Received: 25 March 2020 Accepted: 06 October 2020 Published: 26 October 2020

Citation:

Lu L-F, Li Z-C, Zhang C, Zhou $X-Y$, Zhou Y, Jiang $J-Y$, Chen D-D, Li S and Zhang Y-A (2020) Grass Carp Reovirus (GCRV) Giving Its All to Suppress IFN Production by Countering MAVS Signaling Transduction.

Front. Immunol. 11:545302. doi: 10.3389/fimmu.2020.545302

\section{Grass Carp Reovirus (GCRV) Giving Its All to Suppress IFN Production by Countering MAVS Signaling Transduction}

\author{
Long-Feng Lu ${ }^{1,2 \dagger}$, Zhuo-Cong $\mathrm{Li}^{1,2 \dagger}$, Can Zhang ${ }^{1,2}$, Xiao-Yu Zhou ${ }^{1,3}$, Yu Zhou ${ }^{1,2}$, \\ Jing-Yu Jiang ${ }^{1,2}$, Dan-Dan Chen ${ }^{1,2}$, Shun $\mathrm{Li}^{1,2^{*}}$ and Yong-An Zhang ${ }^{1,4^{*}}$ \\ 1 Institute of Hydrobiology, Chinese Academy of Sciences, Wuhan, China, ${ }^{2}$ College of Advanced Agricultural Sciences, \\ University of Chinese Academy of Sciences, Beijing, China, ${ }^{3}$ College of Fisheries and Life Science, Dalian Ocean University, \\ Dalian, China, ${ }^{4}$ College of Fisheries, Huazhong Agricultural University, Wuhan, China
}

Viruses typically target host RIG-I-like receptors (RLRs), a group of key factors involved in interferon (IFN) production, to enhance viral infection. To date, though immune evasion methods to contradict IFN production have been characterized for a series of terrestrial viruses, the strategies employed by fish viruses remain unclear. Here, we report that all grass carp reovirus (GCRV) proteins encoded by segments $S 1$ to $S 11$ suppress mitochondrial antiviral signaling protein (MAVS)-mediated IFN expression. First, the GCRV viral proteins blunted the MAVS-induced expression of IFN, and impair MAVS antiviral capacity significantly. Interestingly, subsequent co-immunoprecipitation experiments demonstrated that all GCRV viral proteins interacted with several RLR cascades, especially with TANK-binding kinase 1 (TBK1) which was the downstream factor of MAVS. To further illustrate the mechanisms of these interactions between GCRV viral proteins and host RLRs, two of the viral proteins, NS79 (S4) and VP3 (S3), were selected as representative proteins for two distinguished mechanisms. The obtained data demonstrated that NS79 was phosphorylated by gcTBK1, leading to the reduction of host substrate gcIRF3/7 phosphorylation. On the other hand, VP3 degraded gcMAVS and the degradation was significantly reversed by 3-MA. The biological effects of both NS79 and VP3 were consistently found to be related to the suppression of IFN expression and the promotion of viral evasion. Our findings shed light on the special evasion mechanism utilized by fish virus through IFN regulation, which might differ between fish and mammals.

Keywords: GCRV, viral proteins, immune evasion, MAVS, interferon

\section{HIGHLIGHTS}

1. All GCRV proteins encoded by segments S1 to S11 suppress MAVS-mediated IFN expression.

2. GCRV NS79 functions as a decoy substrate for gcTBK1 and reduces the phosphorylation of gCIRF3/7.

3. GCRV VP3 mediates autophagosome-dependent degradation of gcMAVS. 


\section{INTRODUCTION}

Interferons (IFNs) are considered the first and fundamental line of defense against viral invasion in both mammals and fish $(1,2)$. The production of IFNs is triggered by signal transduction once the host cell senses viral components (3). In mammals, IFNs have been divided into three groups as type I $(\alpha, \beta, \omega, \varepsilon$, and $\kappa)$, type II $(\gamma)$, and type III $(\lambda)(4)$. Multiple types of IFNs also identified in fish. The type I IFN genes of zebrafish include IFN $\varphi 1-4$ (5). Salmonids have more IFN genes and there are even 11 genes in Atlantic salmon (6). The current study focuses on the grass carp. There are four homologs of type I IFN in grass carp, termed gcIFN1-gcIFN4. For most viruses, the virion is composed of viral nucleic acids, such as DNA, double stranded RNA (dsRNA), single stranded RNA (ssRNA), and surface glycoproteins (7). Pathogen-associated molecular patterns (PAMPs) (including viral nucleic acids and proteins) are usually recognized by pattern recognition receptors (PRRs), which are expressed on the surface and cytoplasm of host cells (7). Among the PRR members, the retinoic acid inducible gene-I (RIG-I) mediates a pivotal signaling pathway termed the RIG-I-like receptor (RLR) pathway, which significantly activates IFN transcription (8). Upon binding with the viral RNA, RIG-I or melanoma differentiation-associated gene 5 (MDA5) recruits the downstream adaptor mitochondrial antiviral signaling protein (MAVS, also called VISA, IPS-1, or Cardif) (9-12) and the mediator of IFN regulatory factor 3 (IRF3) activation (MITA, also termed STING, MPYS, or ERIS) (13-16), then activates TANK-binding kinase 1 (TBK1). Activated TBK1 further phosphorylates IFN regulatory factor $3 / 7$ (IRF3/7), triggering their dimerization and nuclear translocation to bind to IFN stimulation response elements (ISREs) and initiate the transcription of IFN (17-19).

MAVS is essential for host innate immune responses against viral infection (11). It contains an $\mathrm{N}$-terminal caspase recruitment domain (CARD), a middle proline-rich domain, and a C-terminal transmembrane (TM) domain (20, 21). MAVS is an adaptor protein involved in virus-triggered IFN signaling and regulates virus-induced apoptosis to limit viral replication (22). In fish, multiple-sequence alignments and phylogenetic analysis have demonstrated that teleost fish possess a mavs gene that is involved in the regulation of IFN production (23). For instance, in zebrafish (Danio rerio), MAVS overexpression results in a robust activation and upregulation of IFN and IFNstimulated genes (ISGs) in response to RNA and DNA virus infection (24).

TBK1 is a non-canonical IKB kinase (IKK) that plays critical roles in IFN induction and innate antiviral immunity (25). It consists of three domains: an $\mathrm{N}$-terminal serine/threonine kinase domain (KD), a ubiquitin-like domain (ULD), and a C-terminal domain (CTD) (also known as two C-terminal coiled coil domains) (26). Actually, as a ubiquitously expressed kinase, besides the TBK1IRF3/7 pathway, TBK1 participates in several other signaling pathways such as autophagy and cell cycle control $(27,28)$. TBK1 has been characterized in many fish species, including zebrafish, crucian carp (Carassius auratus), and grass carp
(Ctenopharyngodon idella) (29-31). Overexpression of grass carp TBK1 induces the upregulation of IFN1 upon grass carp reovirus (GCRV) infection (29).

Viruses have evolved elaborate strategies to evade or abrogate the host IFN signaling pathway for their replication. As MAVS and TBK1 are key molecules in the RLR pathway for the activation of IFN production, they are popular targets of viral antagonists. For example, the Newcastle disease virus (NDV) V protein inhibits IFN production through targeting MAVS for ubiquitin-mediated degradation via the E3 ubiquitin ligase RING-finger protein 5 (RNF5) (32). Similarly, the 3C protein of coxsackievirus B3 (CVB3) and porcine reproductive and respiratory syndrome virus (PRRSV) suppresses IFN activation by cleaving MAVS $(33,34)$. The NS3 protein of the hepatitis C virus (HCV) blocks IFN signaling by binding to TBK1 and disrupts the interaction between TBK1 and IRF3 (35). Finally, the leader proteinase (Lbpro) of foot-and-mouth disease virus (FMDV) counteracts host antiviral responses via mediating TBK1 deubiquitination (36). As mentioned above, based on the crucial function of TBK1 on IFN induction, it is the pivotal target for virus for viral immune evasion.

GCRV is a typical pathogen of commercial fishes' viral disease that causes severe epidemic outbreaks of hemorrhagic disease in grass carp, which has an extremely high mortality rate (37). GCRV is a dsRNA virus and belongs to the genus Aquareovirus in the family Reoviridae (38). Based on genomic and biological characteristics, known GCRV strains can be divided into three groups (I-III), and studies have shown that the highest mortality in grass carp is usually caused by group II GCRV (38). GCRV consist of proteins encoded by 11 segments (termed S1-S11) and are encapsulated in multiple layers of icosahedral capsids. Until now, the biological function of these segments encoded proteins is unclear $(39,40)$. In previous studies, fish IFNs and ISGs exhibited a powerful capacity to defend against infection by GCRV $(29,41,42)$. However, GCRV leads to outbreaks of fish hemorrhagic disease (37), indicating that the virus employs a strategy to evade the host IFN response for successful infection. In our previous study, the GCRV VP41 inhibited MITA phosphorylation by acting as a decoy substrate of TBK1, thus reducing IFN production and facilitating viral replication (43). However, GCRV could possess a number of different strategies to elude host defense mechanisms. Therefore, uncovering the other mechanisms used by GCRV to inhibit the activation of IFN signaling is warranted.

In this study, we show that all GCRV viral proteins encoded by $S 1$ to $\mathrm{S} 11$ associate with fish RLR factors, specifically blocking the MAVS-induced IFN expression. Using NS79 and VP3 as representative proteins, we found that NS79 reduces gcMITA phosphorylation by acting as a decoy substrate of gcTBK1 while VP3 degrades gcMAVS in an autophagosome-dependent manner, ultimately blocking IFN production and facilitating virus replication. These results uncovered two distinct evasion strategies used by GCRV to escape the host IFN system by targeting gcRLR factors. Our findings will lay the foundation for further study of the crosstalk between the host IFN response and viral infection in fish species. 


\section{MATERIALS AND METHODS}

\section{Cells and Viruses}

Human embryonic kidney (HEK) 293T cells were provided by Dr. Xing Liu (Institute of Hydrobiology, Chinese Academy of Sciences) and were grown at $37^{\circ} \mathrm{C}$ in $5 \% \mathrm{CO} 2$ in Dulbecco's modified Eagle's medium (DMEM; Invitrogen) supplemented with $10 \%$ fetal bovine serum (FBS, Invitrogen). Grass carp ovary (GCO) cells and Epithelioma papulosum cyprini (EPC) cells were obtained from China Center for Type Culture Collection (CCTCC) and were maintained at $28^{\circ} \mathrm{C}$ in $5 \% \mathrm{CO} 2$ in medium 199 (Invitrogen) supplemented with 10\% FBS. GCRV (strain 106, group II) was a gift from Lingbing Zeng (Yangtze River Fisheries Research Institute, Chinese Academy of Fishery Sciences). Because group II GCRV cannot cause a cytopathic effect (CPE) but can propagate in GCO cells, the cultured media with GCO cells infected with group II GCRV for 8 days were harvested and stored at $-80^{\circ} \mathrm{C}$ until used. Spring viremia of carp virus (SVCV), a negative ssRNA virus, was propagated in EPC cells until CPE was observed; then the harvested cell culture fluid containing SVCV was centrifuged at $4 \times 10^{3} \mathrm{~g}$ for $20 \mathrm{~min}$ to remove the cell debris, and the supernatant was stored at $-80^{\circ} \mathrm{C}$ until used.

\section{Plasmid Construction and Reagents}

The open reading frame (ORF) of GCRV S1-S11 (KC201166.1, KC201167.1, KC201168.1, KC201169.1, KC201170.1, KC201171.1, KC201172.1, KC201173.1, KC201174.1, KC201175.1, KC201176.1) were generated by PCR and then cloned into pcDNA3.1(+) (Invitrogen), pCMV-Myc (Clontech), or pCMV-HA vectors (Clontech), respectively. The ORFs of gcRIG-I (GQ478334.2), gcMAVS (KF366908.1), gcTBK1 (JN704345.1), gcMITA (JN786909.1), gcIRF3 (KT347289.1), and gcIRF7 (KY613780.1) were also subcloned into pcDNA3.1 (+), pCMV-Myc, pCMV-HA, and pCMV-Tag2C vectors, respectively. The ORF of glyceraldehyde-3-phosphate dehydrogenase (GAPDH) (NM_001115114.1) was subcloned into pCMV-Myc and pCMV-Tag2C vectors, respectively. For subcellular localization, the ORFs of VP3, NS79, and zebrafish LC3 (NM_199604.1) were inserted into pEGFP-N3 vector (Clontech), respectively. The ORFs of gcMAVS and gcTBK1 were also inserted into pCS2-mCherry vector (Clontech). The expression plasmids for Flag/pcDNA3.1-DrMAVS, Flag/ pcDNA3.1-DrTBK1, Flag-DrMITA, Flag-DrIRF3, and FlagDrIRF7 were described previously (44). For promoter activity analysis, gcIFN1/gcIFN2/gcIFN3/gcIFN4pro-Luc construct were generated by insertion of corresponding 5 '-flanking regulatory region of gcIFN1 promoter (GU139255.1), gcIFN2 promoter (KY613781.1), gcIFN3 promoter (KY613782.1), or gcIFN4 promoter (KY613783.1) into pGL3-basic luciferase reporter vector (Promega, Madison, WI), respectively. The DrIFN $\varphi 1$ pro-Luc and ISRE-Luc plasmids in the pGL3-basic luciferase reporter vector (Promega) were constructed as described previously (45). The Renilla luciferase internal control vector ( $\mathrm{pRL}-\mathrm{TK}$ ) was purchased from Promega. The primers including the restriction enzyme cutting sites used for plasmid construction are listed in Supplemental Table I. All constructs were confirmed by DNA sequencing.

\section{Luciferase Activity Assay}

EPC cells or GCO cells $\left(\sim 2 \times 10^{4}\right.$ cells $)$ were seeded in 24-well plates overnight and co-transfected with the indicated luciferase reporter plasmid and overexpression plasmid. The empty vector pcDNA3.1(+) was used to ensure equivalent amounts of total DNA in each well. Transfection of $1 \mu \mathrm{g} / \mathrm{ml}$ poly I:C (SigmaAldrich, P1530) by using FishTrans (MeiSenTe Biotechnology) was performed at $24 \mathrm{~h}$ before cell harvest. At $48 \mathrm{~h}$ posttransfection, the cells were washed with phosphate-buffered saline (PBS) and lysed for measuring luciferase activity by the Dual-Luciferase Reporter Assay System (Promega) according to the manufacturer's instructions. Firefly luciferase activity was normalized on the basis of Renilla luciferase activity.

\section{RNA Extraction, Reverse Transcription, and $\mathrm{qPCR}$}

Total RNA was extracted by the TRIzol reagent (Invitrogen). First-strand cDNA was synthesized by using a GoScript reverse transcription system (Promega) according to the manufacturer's instructions. qPCR was performed with Fast SYBR green PCR master mix (Bio-Rad) on the CFX96 real-time system (Bio-Rad). PCR conditions were as follows: $95^{\circ} \mathrm{C}$ for $5 \mathrm{~min}$ and then 40 cycles of $95^{\circ} \mathrm{C}$ for $20 \mathrm{~s}, 60^{\circ} \mathrm{C}$ for $20 \mathrm{~s}$, and $72^{\circ} \mathrm{C}$ for $20 \mathrm{~s}$. All primers used for qPCR are shown in Supplemental Table I, and the $\beta$-actin gene was used as an internal control. The relative fold changes were calculated by comparison to the corresponding controls using the $2^{-\Delta \Delta \mathrm{Ct}}$ method.

\section{Transient Transfection and Virus Infection}

Transient transfections were performed in EPC cells seeded in 6well $\left(\sim 1.5 \times 10^{5}\right.$ cells $)$ or 24 -well plates $\left(\sim 2 \times 10^{4}\right.$ cells $)$ by using FishTrans DNA Transfection Reagent according to the manufacturer's protocol. For the antiviral assay using 24-well plates, EPC cells were transfected with $0.5 \mu \mathrm{g}$ pcDNA3.1-VP3/ NS79 or the empty vector. At 24 h post-transfection, cells were infected with SVCV at a multiplicity of infection $(\mathrm{MOI}=0.001)$. After $48 \mathrm{~h}$ or $72 \mathrm{~h}$, supernatant aliquots were harvested for detection of virus titers, the cell monolayers were fixed by $4 \%$ paraformaldehyde (PFA) and stained with $1 \%$ crystal violet for visualizing $\mathrm{CPE}$. For virus titration, $200 \mu \mathrm{l}$ of culture medium were collected at $48 \mathrm{~h}$ post-infection, and used for detection of virus titers according to the method of Reed and Muench (29). The supernatants were subjected to 3-fold serial dilutions and then added $(100 \mu \mathrm{l})$ onto a monolayer of EPC cells cultured in a 96-well plate $\left(\sim 3 \times 10^{3}\right.$ cells). After 48 or $72 \mathrm{~h}$, the medium was removed and the cells were washed with PBS, fixed by $4 \%$ PFA and stained with $1 \%$ crystal violet. The virus titer was expressed as $50 \%$ tissue culture infective dose $\left(\mathrm{TCID}_{50} / \mathrm{ml}\right)$.

\section{Co-immunoprecipitation (Co-IP) Assay}

For Co-IP experiments, HEK 293T cells were used instead of EPC cells (transfection efficiency approximately 30\%) due to the 
superhigh transfection efficiency of HEK 293T cells (90\%). Cells seeded in $10 \mathrm{~cm}^{2}$ dishes $\left(\sim 6 \times 10^{6}\right.$ cells $)$ overnight were transfected with a total of $10 \mu \mathrm{g}$ of the plasmids indicated on the figures. At $24 \mathrm{~h}$ post-transfection, the medium was removed carefully, and the cell monolayer was washed twice with $10 \mathrm{ml}$ ice-cold PBS. Then the cells were lysed in $1 \mathrm{ml}$ of radioimmunoprecipitation (RIPA) lysis buffer [1\% NP-40, 50 mM Tris-HCl, pH 7.5, 150 mM NaCl, 1 mM EDTA, 1 mM NaF, $1 \mathrm{mM}$ sodium orthovanadate (Na3VO4), $1 \mathrm{mM}$ phenylmethylsulfonyl fluoride (PMSF), $0.25 \%$ sodium deoxycholate] containing protease inhibitor cocktail (1\%, Sigma-Aldrich) at $4^{\circ} \mathrm{C}$ for $1 \mathrm{~h}$ on a rocker platform. The cellular debris was removed by centrifugation at $12,000 \times \mathrm{g}$ for $15 \mathrm{~min}$ at $4^{\circ} \mathrm{C}$. The supernatant was transferred to a fresh tube and incubated with $30 \mu \mathrm{l}$ anti-HA-agarose beads or anti-Flag/Myc affinity gel (Sigma-Aldrich) overnight at $4^{\circ} \mathrm{C}$ with constant agitation. Immunoprecipitated proteins were collected by centrifugation at 5,000 $\times \mathrm{g}$ for $1 \mathrm{~min}$ at $4^{\circ} \mathrm{C}$, washed three times with lysis buffer and resuspended in $50 \mu \mathrm{l} 2 \times$ SDS sample buffer. The immunoprecipitates and whole cell lysates were analyzed by IB with the indicated antibodies (Abs).

\section{Immunoblot Analysis}

Immunoprecipitates or whole cell lysates were separated by $10 \%$ SDS-PAGE and transferred to polyvinylidene difluoride (PVDF) membrane (Trans-Blot Turbo ${ }^{\mathrm{TM}}$ Transfer System, Bio-Rad). The membranes were blocked for $1 \mathrm{~h}$ at room temperature in TBST buffer (25 mM Tris-HCl, $150 \mathrm{mM} \mathrm{NaCl}, 0.1 \%$ Tween 20, pH 7.5) containing $5 \%$ nonfat dry milk, probed with the indicated primary Abs at an appropriate dilution overnight at $4^{\circ} \mathrm{C}$, washed three times with TBST, and then incubated with secondary Abs for $1 \mathrm{~h}$ at room temperature. After three additional washes with TBST, the membranes were stained with the Immobilon Western chemiluminescent horseradish peroxidase (HRP) substrate (Millipore) and detected by using an ImageQuant LAS 4000 system (GE Healthcare). Abs were diluted as follows: anti- $\beta$-actin (Cell Signaling Technology) at 1:1,000, anti-Flag/HA (Sigma-Aldrich) at 1:3,000, anti-Myc (Santa Cruz Biotechnology) at 1:2,000, and HRP-conjugated anti-mouse IgG (Thermo Scientific) at 1:5,000. Results are representative of three independent experiments.

\section{In Vitro Protein Dephosphorylation Assay}

Transfected GCO cells were lysed as described above, except that the phosphatase inhibitors (Na3VO4 and EDTA) were omitted from the lysis buffer. Protein dephosphorylation was carried out in $100 \mu \mathrm{l}$ reaction mixtures consisting of $100 \mu \mathrm{g}$ of cell protein and 10 units (U) of calf intestinal phosphatase (CIP) (SigmaAldrich). The reaction mixtures were incubated at $37^{\circ} \mathrm{C}$ for $40 \mathrm{~min}$, followed by immunoblot analysis.

\section{Fluorescent Microscopy}

EPC cells were plated onto coverslips in 6-well plates $\left(\sim 6 \times 10^{4}\right.$ cells) and transfected with the plasmids indicated on the figures for $24 \mathrm{~h}$. Then the cells were washed twice with PBS and fixed with $4 \%$ PFA for $1 \mathrm{~h}$. After being washed three times with PBS, the cells were stained with $1 \mu \mathrm{g} / \mathrm{ml} \mathrm{4}$, 6-diamidino-2- phenylindole (DAPI; Beyotime) for $15 \mathrm{~min}$ in the dark at room temperature. Finally, the coverslips were washed and observed with a confocal microscope under a $63 \times$ oil immersion objective (SP8; Leica).

\section{Statistics Analysis}

Luciferase, qPCR, and virus titer detection data are expressed as the mean \pm standard error of the mean (SEM) $(n \geq 3)$. The $\mathrm{p}$ values were calculated by the Student's t-test or one-way analysis of variance (ANOVA) with Dunnett's post hoc test (SPSS Statistics, version 19; IBM). A $p$ value $<0.05$ was considered statistically significant.

\section{RESULTS}

\section{The MAVS-Mediated IFN Activation Is Blocked by All Viral Proteins of GCRV}

To date, the role of gcIFN1-gcIFN4 under influence remain unclear; therefore, the characterization of these IFNs was first performed. Treatment with poly I:C, a synthetic dsRNA molecule that is a potent inducer of type I IFNs, resulted in a significant increase in gcIFN1 promoter (gcIFN1pro) activity compared with other IFNs (Figure 1A). As fish RLR cascades are pivotal IFN activators, the upstream factors gcRIG-I, gcMAVS, and gcTBK1 were employed for IFN identification. Consistent with the above result, gcIFN1 displayed remarkable activation under gcRIG-I, gcMAVS, and gcTBK1 stimulation (Figures 1BD). Therefore, gcIFN1 was selected as the reporter gene for subsequent assays.

The 11 segments of GCRV were subcloned into eukaryotic expression vectors to investigate the roles of GCRV viral proteins in IFN regulation. As shown in Figure 1E, poly I:C stimulation significantly induced gcIFN1pro activation, whereas this induction was inhibited by all GCRV viral proteins to varying degrees. ISRE is considered as a transcription factor-binding motif in the promoter regions of IFNs and ISGs, after transfection with plasmids encoding GCRV S1-S11 and ISRELuc and stimulation with poly I:C, the activation of ISRE was also suppressed by all GCRV viral proteins (Figure 1F). Subsequently, MAVS-mediated innate immune signaling is critical to the activation of IFN expression. Thus, whether GCRV viral proteins involved in MAVS-mediated IFN induction was investigated. Interestingly, all 11 viral proteins repressed the gcMAVS-induced gcIFN1 and ISRE activity (Figures 1G, H). Next, we examined whether GCRV viral proteins had an effect on the gcMAVS-mediated antiviral response. We transfected EPC cells with gcMAVS, together with a control plasmid or S1-S11 expression plasmids. Then transfected cells were infected with SVCV. As shown in Figure 1I, stronger CPEs were observed in the GCRV viral proteins groups at $48 \mathrm{~h}$ post-infection. These were confirmed by the titer of SVCV, overexpression of gcMAVS decreased the viral titer 2,700-fold compared to that in control cells, whereas viral productions in GCRV viral proteins-expressing cells were increased compared with that in the gcMAVS-overexpressed 
A

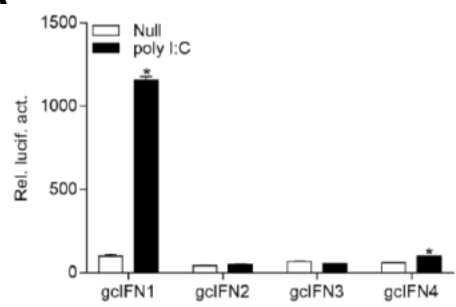

E

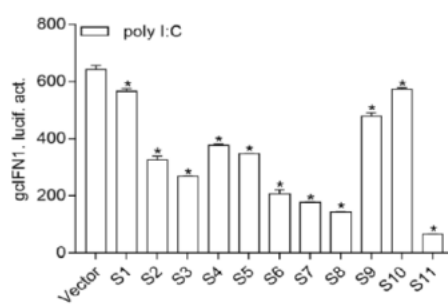

I

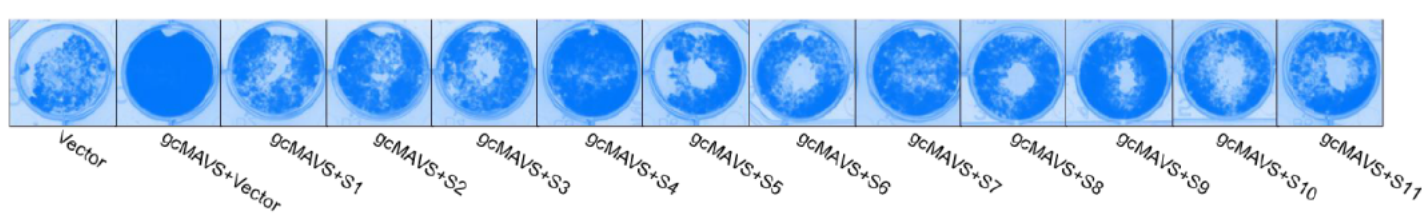

B

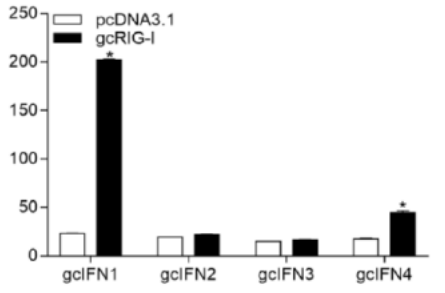

F

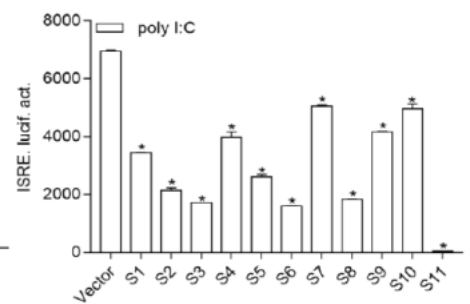

G

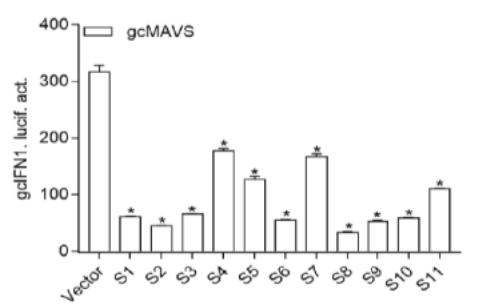

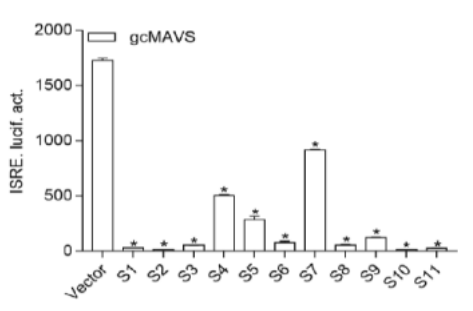

$\mathbf{J}$

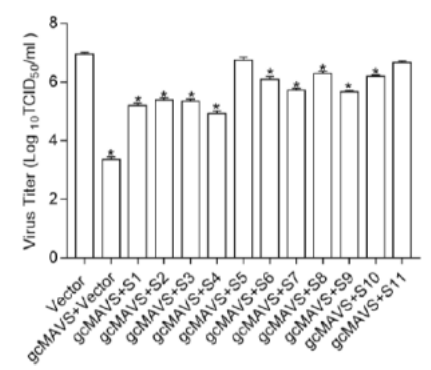

FIGURE 1 | GCRV viral proteins encoded by S1-S11 suppress the MAVS-mediated activation of IFN1 and its antiviral effect. (A) Induction of gcIFN1, gclFN2, gclFN3, gclFN4 promoters by poly I:C. GCO cells were seeded in 24-well plates overnight were transfected with $0.25 \mu \mathrm{g}$ gclFN1pro-Luc, gclFN2pro-Luc, gclFN3pro-Luc, or gclFN4pro-Luc, $50 \mathrm{ng}$ pRL-TK was used as an internal control. At $24 \mathrm{~h}$ post-transfection, cells were transfected with poly l:C $(1 \mu \mathrm{g} / \mathrm{ml})$ or left untreated (null). The luciferase assay was performed $24 \mathrm{~h}$ after stimulation. (B-D) Activation of gclFN1, gclFN2, gclFN3, gclFN4 by overexpression of gcRIG-I or gcMAVS or gcTBK1. GCO cells seeded in 24-well plates overnight were co-transfected with pcDNA3.1-gcRIG-I (B) or pcDNA3.1-gcMAVS (C) or pcDNA3.1-gcTBK1 (D) or empty vector and gcIFN1pro-Luc, gcIFN2pro-Luc, gcIFN3pro-Luc, or gclFN4pro-Luc at the ratio of 1:1. pRL-TK was used as a control. Luciferase activities were analyzed at $24 \mathrm{~h}$ post-transfection. (E, F) GCRV viral proteins suppresses poly I:Cinduced gcIFN1 and ISRE activation. GCO cells were seeded in 24-well plates and co-transfected with $0.25 \mu \mathrm{g}$ empty vector or pcDNA3.1-S1, or pcDNA3.1-S2, or pcDNA3.1-S3, or pcDNA3.1-S4, or pCDNA3.1S5, or pcDNA3.1-S6, or pcDNA3.1-S7, or pcDNA3.1-S8, or pcDNA3.1-S9, or pcDNA3.1-S10, or pcDNA3.1-S11, plus 0.25 $\mu$ g gclFN1 pro-Luc (E) or ISRE-Luc (F), 50 ng pRL-TK was used as a control. At 24 $\mathrm{h}$ post-transfection, cells were transfected with poly l:C $(1 \mu \mathrm{g} / \mathrm{mll})$. The luciferase assay was performed $24 \mathrm{~h}$ after stimulation. (G, H) GCRV viral proteins inhibit gclFN1 and ISRE activation mediated by gcMAVS. GCO cells were seeded in 24-well plates and co-transfected with pcDNA3.1-gcMAVS and empty vector or pcDNA3.1-S1, or pcDNA3.1-S2, or pcDNA3.1-S3, or pcDNA3.1-S4, or pcDNA3.1-S5, or pcDNA3.1-S6, or pcDNA3.1-S7, or pcDNA3.1-S8, or pcDNA3.1-S9, or pcDNA3.1-S10, or pcDNA3.1-S11, plus gclFN1pro-Luc (G) or ISRE-Luc (H) at the ratio of 1:1:1. pRL-TK was used as a control. At 24 h post-transfection, cells were lysed for luciferase activity detection. The promoter activity is presented as relative light units normalized to Renilla luciferase activity. Data were expressed as mean \pm SEM, $n=3$. Asterisks indicate significant differences from control $\left({ }^{*} p<0.05\right)$. (I) The S1-S11 segments inhibit the antiviral effect of gcMAVS. EPC cells were seeded in 24-well plates overnight and transfected with indicated plasmids for $24 \mathrm{~h}$, then the cells were infected with SVCV (MOI = 0.001) for $48 \mathrm{~h}$. (I) Then, cells were fixed with $4 \%$ PFA and stained with $1 \%$ crystal violet. (J) Culture supernatants from the cells infected with SVCV were collected, and the viral titer was measured according to the method of Reed and Muench. 
cells (Figure 1J). These results demonstrate that the GCRV viral proteins impaired MAVS-induced activation of IFN and inhibited the MAVS-mediated antiviral response.

\section{TBK1, Which Is Downstream of MAVS, Is the Common Target of GCRV Viral Proteins}

Combined with the observation that MAVS recruits TBK1 in the signaling transduction process, the above results suggest that the GCRV viral proteins block MAVS-mediated IFN activation. Thus, co-IP experiments were performed to characterize the relationship between the GCRV viral proteins and gcTBK1. When Myc-tagged GCRV S1 to S11 and Flag-tagged gcTBK1 were overexpressed, the anti-Myc Ab-immunoprecipitated S1 to S11 protein complexes were recognized by the anti-Flag Ab (Figure 2A), and vice versa (Figures 2B-L), demonstrating that all GCRV viral proteins were associated with grass carp TBK1. The results showed that all GCRV viral proteins interacted with the RLR molecules, particularly with TBK1. To our knowledge, these data manifest a novel mechanism that all GCRV viral proteins may associate with TBK1, which is the key factor in IFN activation.

\section{GCRV NS79 Suppresses IFN Induction and Is phosphorylated by gcTBK1}

The above observations revealed that all GCRV viral proteins blunt MAVS-induced IFN activation. To further investigate the specific mechanisms underlying how GCRV proteins evade IFN responses, we chose S3 and S4-encoded proteins for the subsequent assays. The S3 and S4 segments of group II GCRV were predicted to encode the respective inner core protein VP3 and the nonstructural protein NS79, which are involved in viral inclusion body formation (46). As shown in Figure 3A, poly I:C stimulation induced the activation of gcIFN1pro and ISRE; however, this induction was significantly blocked by the overexpression of NS79. In addition, overexpression of gcMAVS led to a significant induction of gcIFN1pro or ISRE activity, whereas it was inhibited by co-transfection with NS79 (Figure 3B). To further explore the function of NS79, its subcellular locations were monitored. EPC cells were cotransfected mCherry-gcMAVS or mCherry-gcTBK1 with EGFP-NS79. Red signals from gcMAVS and gcTBK1 were observed in the cytosol and overlapped with the green signals from NS79 (Figures 3C, D). These data suggest that GCRV NS79 was colocalized with gcMAVS and gcTBK1 in the cytosol.

To further probe the regulatory mechanism of NS79 on the RLR axis, we analyzed the effect of NS79 on RLR molecules at the protein level. gcMAVS-, gcTBK1-, gcMITA-, gcIRF3-, and gcIRF7-Myc expression vectors were co-transfected with HANS79 or an empty vector. As shown in Figure 3E, NS79 had no apparent effects on the RLR factors at the protein level. However, when Myc-NS79 was co-transfected with Flag-gcTBK1, shift bands with higher molecular weights were observed. One possible reason for this observation is that NS79 can be phosphorylated by gcTBK1. To confirm this hypothesis, a dephosphorylation assay was performed in vitro. As expected, the shift bands partially disappeared after treatment with CIP, indicating that GCRV NS79 can be phosphorylated by gcTBK1 (Figure 3F). Given that the N-terminal domain is the functional kinase domain for TBK1, the truncated mutant of gcTBK1 was constructed to identify the functional domain on NS79. As shown in Figure 3G, compared to the abundant NS79 phosphorylation found in the wild-type gcTBK1 group, gcTBK1- $\Delta \mathrm{N}$ (lacking the $\mathrm{N}$ terminus) failed to phosphorylate NS79. These data demonstrate that NS79 blocks IFN production and is phosphorylated by gcTBK1 via its N-terminal domain.

\section{NS79 Decreases gcTBK1-Mediated Phosphorylation of gclRF3}

To further determine the biological effect of NS79 on gcTBK1mediated signaling responses, the functions of gcTBK1 were investigated. As shown in Figures 4A-C, co-transfection with Flag-gcTBK1 caused a shift of gcMITA, gcIRF3, or gcIRF7 to higher-molecular-weight bands. Subsequently, after the cell lysates were incubated with CIP, the shift bands disappeared, indicating that gcMITA, gcIRF3, and gcIRF7 are also phosphorylated by gcTBK1 in grass carp. Furthermore, the truncated mutant of gcTBK1 was used to characterize the functional kinase domain of gcTBK1. Compared with the wildtype gcTBK1, gcTBK1- $\Delta \mathrm{N}$ was unable to phosphorylate gcMITA, gcIRF3, or gcIRF7 (Figures 4D-F). These data suggest that the $\mathrm{N}$-terminal kinase domain is also essential for gcMITA, gcIRF3, and gcIRF7 phosphorylation.

Next, we wondered whether NS79 affects the gcTBK1induced phosphorylation of gcMITA, gcIRF3, and gcIRF7. As shown in Figures 4G-I, the bands of gcMITA, gcIRF3, gcIRF7, and NS79 exhibited higher mobility when the cells were cotransfected with Flag-gcTBK1, but the phosphorylated gcMITA, gcIRF3, and gcIRF7 were reduced when co-transfected with NS79. In conclusion, these results indicate that the GCRV NS79 reduces the gcTBK1-triggered phosphorylation of gcMITA, gcIRF3, and gcIRF7 by being competitively phosphorylated by gcTBK1.

\section{GCRV VP3 Mediates Autophagosome- Dependent Degradation of gcMAVS}

The capacity of another GCRV viral protein VP3 on IFN expression was explored as well. As shown in Figure 5A, the activation of gcIFN1pro and ISRE induced by poly I:C were markedly impaired in VP3 overexpressing cells. In addition, VP3 also impeded the MAVS-induced gcIFN1pro and ISRE activation (Figure 5B). Combining with results of inhibition with gcMAVS and interaction with gcTBK1 of VP3, the subcellular locations of VP3 and gcMAVS or gcTBK1 were determined. gcMAVS-, gcTBK1-mCherry plasmids, and EGFPVP3 were co-transfected into EPC cells, and the red signals from gcMAVS and gcTBK1 were observed in the cytosol and almost overlapped with the green signals from VP3 (Figures 5C, D).

Furthermore, the specific signaling molecule targeted by VP3 was investigated different signaling molecules were co-expressed with HA-VP3 in EPC cells and found that the abundance of gcMAVS was substantially decreased with the overexpression of 
A

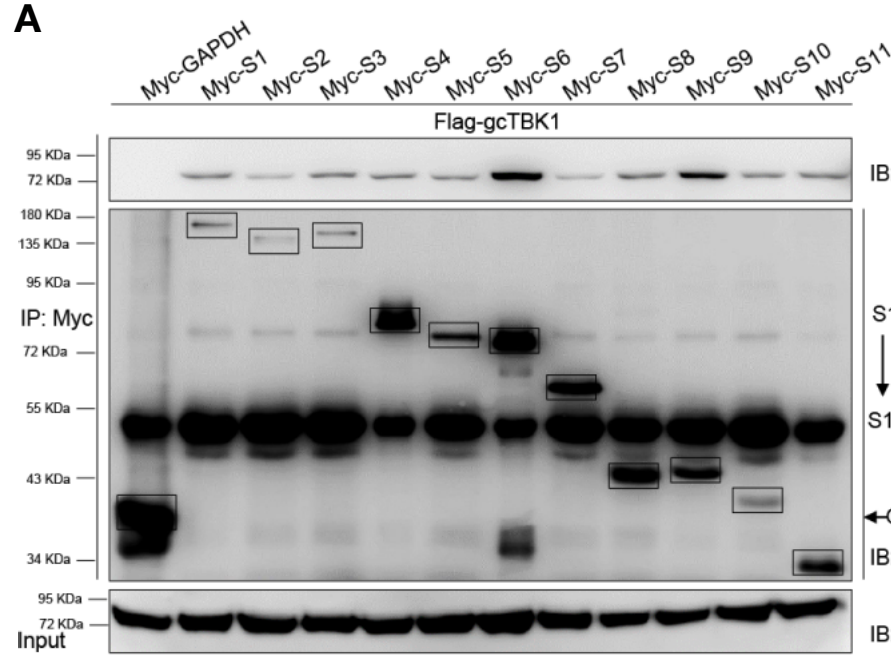

H

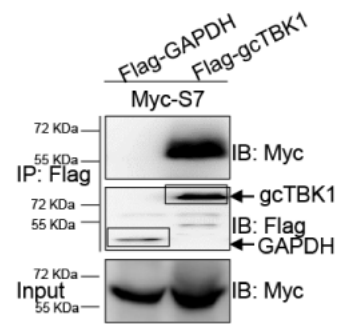

B

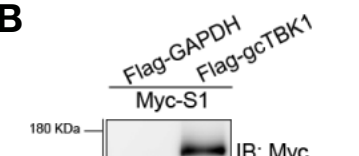
IB: Flag IP: Flag $72 \mathrm{KDa}$
$55 \mathrm{KDa}$

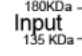

E

\section{E}

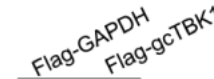
$95 \mathrm{KDa}$

IB: Myc IB: Myc $\quad{ }_{55}^{72 \mathrm{KDa}}$ IB: Flag $\begin{gathered}95 \mathrm{KDa}- \\ \text { Input } \\ 72 \mathrm{KDa}\end{gathered}-$ IB: Myc
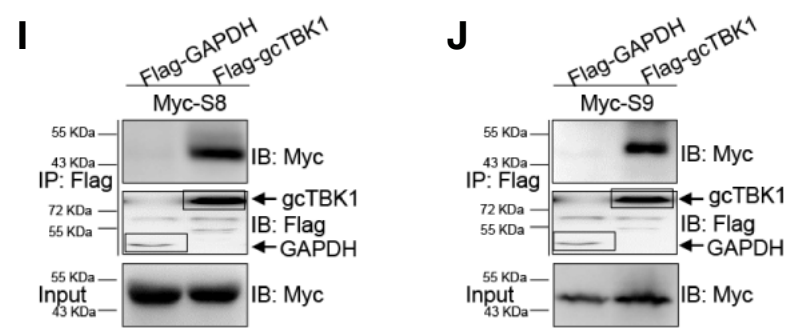

C
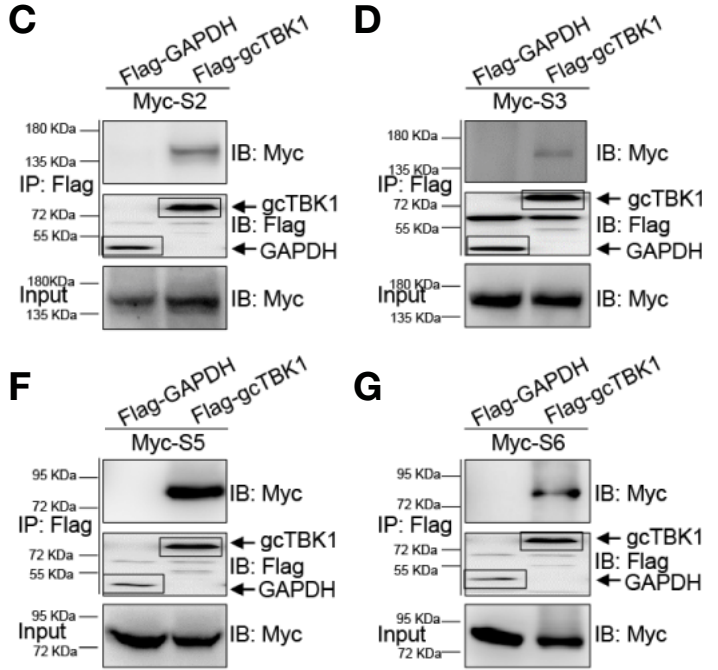

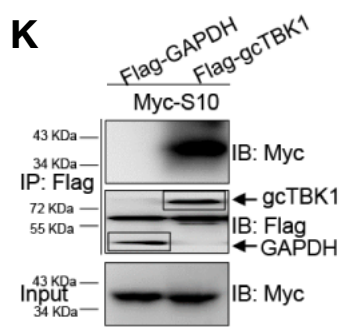

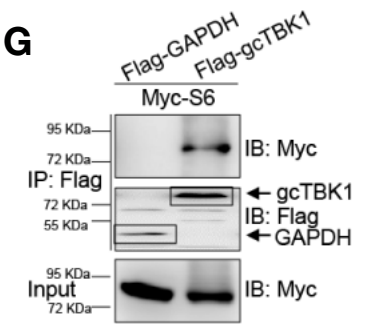

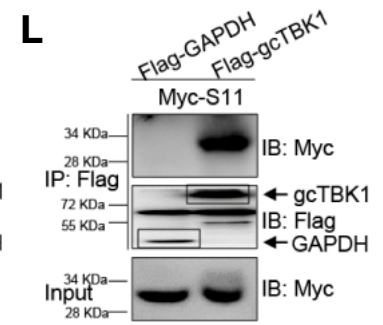

FIGURE 2 | The interaction between GCRV viral proteins and gcTBK1. (A-L) HEK 293T cells seeded in 10-cm² dishes were transfected with the indicated plasmids (5 $\mu$ g each). After 24 h, cell lysates were immunoprecipitated (IP) with an anti-Myc/Flag affinity gel. Then the immunoprecipitates and cell lysates were analyzed by immunoblotting (IB) with the anti-Flag and anti-Myc Abs, respectively. All experiments were repeated at least three times, and with similar results. 
A
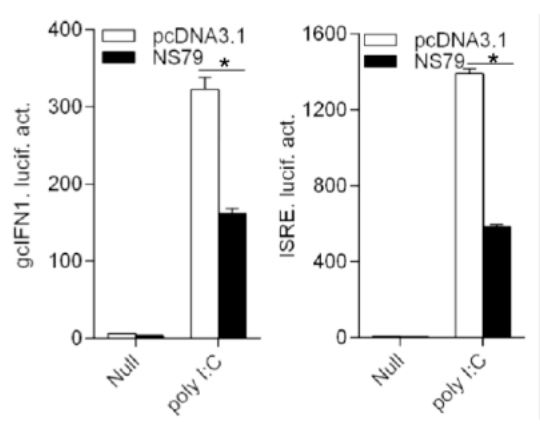

B

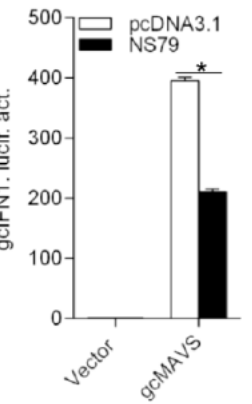

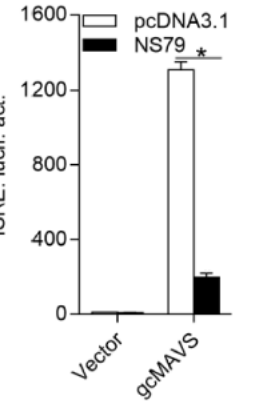

D
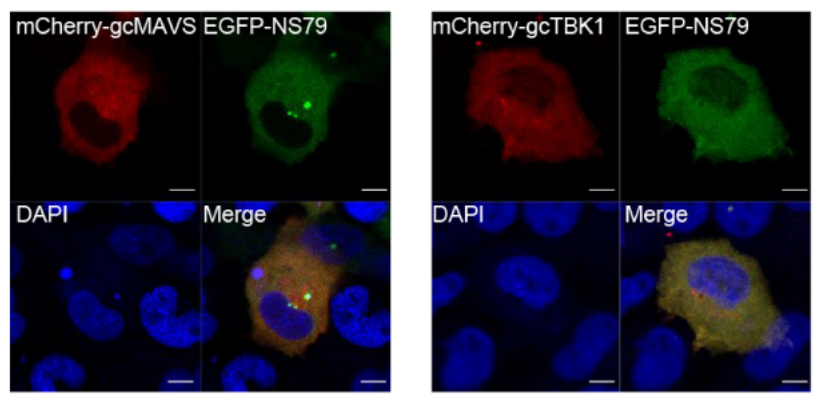

$\mathbf{F}$
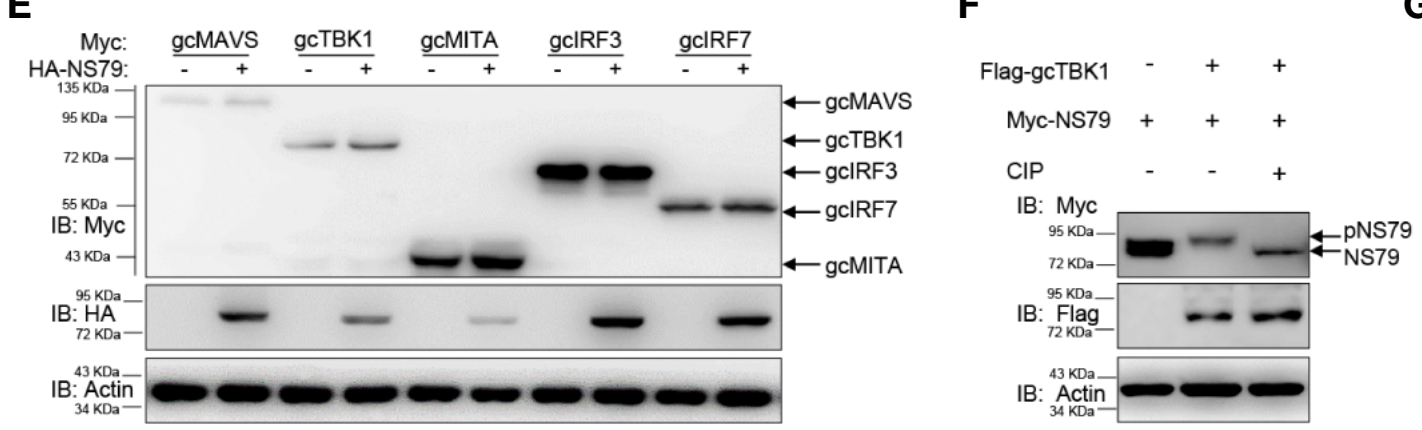

G

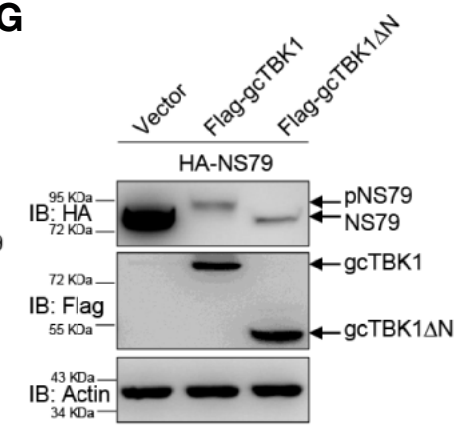

FIGURE 3 | GCRV NS79 inhibits gcMAVS-induced gcIFN1 and ISRE activation and is phosphorylated by the gcTBK1 N terminus. (A) Overexpression of NS79 inhibits poly I:C-induced gcIFN1pro/ISRE activation. EPC cells were seeded in 24-well plates overnight and co-transfected with $0.25 \mu \mathrm{g}$ gcIFN1pro-Luc or ISRE-Luc, and $50 \mathrm{ng}$ pRL-TK, plus $0.25 \mu \mathrm{g}$ empty vector or pcDNA3.1-NS79. At $24 \mathrm{~h}$ post- transfection, cells were transfected with poly I:C $(1 \mu \mathrm{g} / \mathrm{ml})$ or left untreated (null). The luciferase assay was performed $24 \mathrm{~h}$ after stimulation. (B) NS79 suppresses gclFN1pro/ISRE activation mediated by gcMAVS. EPC cells were seeded in 24-well plates and co-transfected with gcMAVS-expressing plasmids and empty vector or pcDNA3.1-NS79, plus gcIFN1pro-Luc or ISRE-Luc at the ratio of 1:1:1. pRL-TK was used as a control. At 24 $\mathrm{h}$ post-transfection, cells were lysed for luciferase activity detection. The promoter activity is presented as relative light units normalized to Renilla luciferase activity. Data were expressed as mean $\pm \mathrm{SEM}, n=3$.

Asterisks indicate significant differences from control ( ${ }^{*} p<0.05$ ). (C, D) EPC cells were seeded on microscopy cover glass in 6-well plates and cotransfected with $2 \mu \mathrm{g}$ EGFP-NS79 and $2 \mu \mathrm{g}$ mCherry-gCMAVS (C) or mCherry-gcTBK1 (D). After $24 \mathrm{~h}$, the cells were fixed and subjected to confocal microscopy analysis. Green signals represent overexpressed NS79, red signals represent overexpressed gcMAVS or gcTBK1, and blue staining indicates the nucleus region. (original magnification, 63× oil immersion objective). Scale bar, $10 \mu \mathrm{m}$. (E) NS79 has no effect on the exogenous gcRLR factors. EPC cells were seeded in 6-well plates overnight and transfected with the indicated plasmids (1 $\mu \mathrm{g}$ each) for $24 \mathrm{~h}$. The cell lysates were subjected to IB with anti-Myc, anti-HA, and anti- $\beta$-actin Abs. (F) gcTBK1 mediates the phosphorylation of NS79. GCO cells were seeded into 6-well plates overnight and transfected with the indicated plasmids ( $1.5 \mu \mathrm{g}$ each) for $24 \mathrm{~h}$. The cell lysates (100 $\mu \mathrm{g})$ were treated with or without $\mathrm{CIP}(10 \mathrm{U})$ for $40 \mathrm{~min}$ at $37^{\circ} \mathrm{C}$. Then the lysates were detected by IB with anti-Myc, anti-Flag, and anti- $\beta$-actin Abs. (G) The N-terminal kinase domain of gcTBK1 is the functional region that phosphorylates NS79. GCO cells were seeded into 6 -well plates overnight and transfected with the indicated plasmids ( $1.5 \mu \mathrm{g}$ each) for $24 \mathrm{~h}$. The cell lysates were subjected to IB with anti-HA, anti-Flag, and anti- $\beta$-actin Abs. 

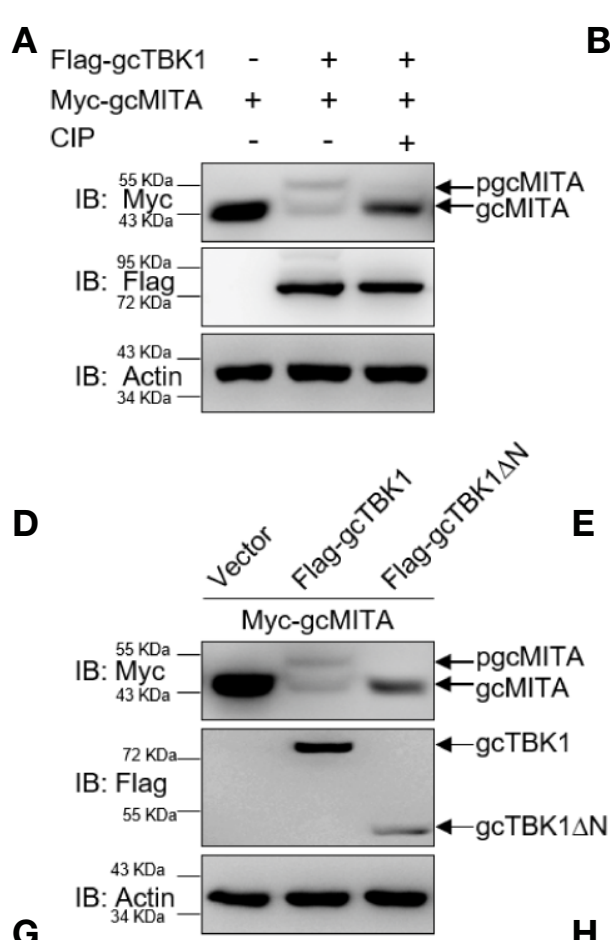

G

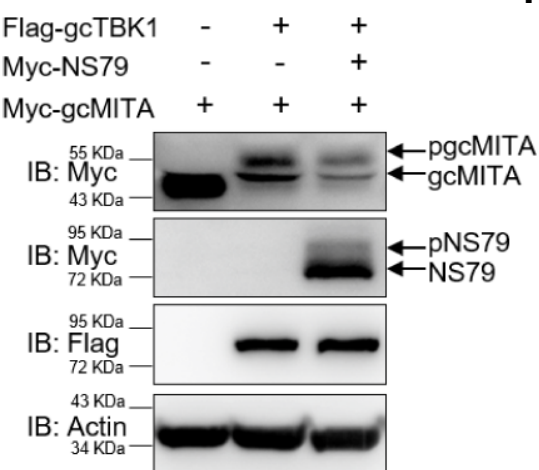

B

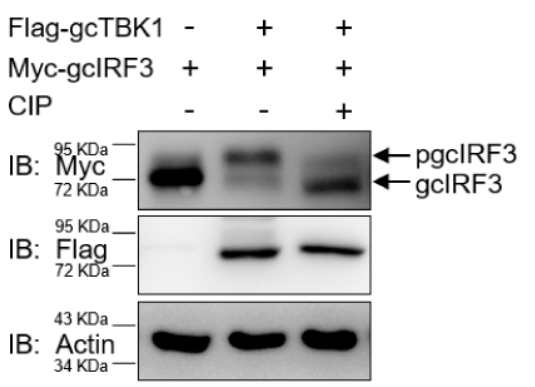

E

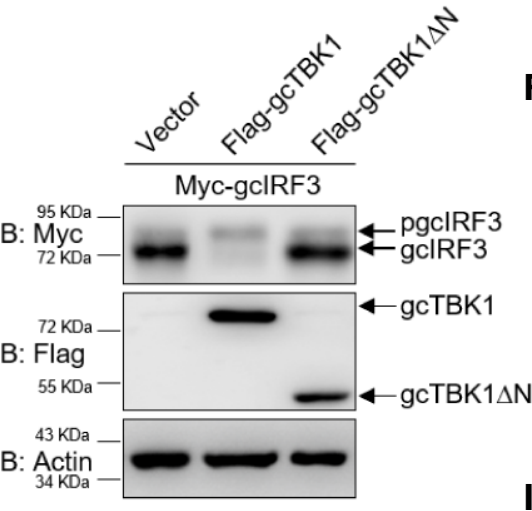

Flag-gcTBK1 - $+\quad+$

Myc-NS79 - $\quad+$

Myc-gclRF3 +++

IB: $\underset{72 \mathrm{KDa}}{95 \mathrm{KDa}}-\square=\longleftarrow_{\mathrm{gclRF} 3}^{\mathrm{pgclRF} 3}$

$95 \mathrm{KDa}-\mathrm{PNS79}$
$\mathrm{M}_{72 \mathrm{KDa}}-\mathrm{NS79}$

IB: $\stackrel{95 \mathrm{KDa}}{\text { Flag }}$

rera

B: Actin
C Flag-gcTBK1 - ++

Myc-gclRF7 +++

CIP $\quad-\quad-\quad+$

IB: $\mathrm{MyC}_{55 \mathrm{KDa}}^{72 \mathrm{KDa}}-\longrightarrow$

IB: $\mathrm{Flag}_{72}^{95 \mathrm{KDa}}-\longrightarrow$

B: Actin

$\mathbf{F}$

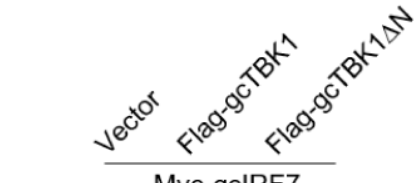

IB: Myc ${ }_{55 \mathrm{KDa}}^{72 \mathrm{KDa}}=\underset{\text { Myc-gcIRF7 }}{\longleftarrow \text { gclRF7 }}$

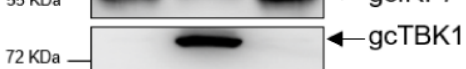

IB: Flag

$55 \mathrm{KDa}-\longrightarrow$ gcTBK $1 \Delta \mathrm{N}$

IB: Actin

$\mathrm{Actin}_{34 \mathrm{KDa}}$

Flag-gcTBK1 - ++

Myc-NS79 - - +

Myc-gclRF7 + $++\quad$

IB: $\mathrm{Myc}_{55 \mathrm{KDa}}^{72 \mathrm{KDa}}-\underset{\mathrm{gclRF}}{\longleftarrow}$

IB: $\begin{gathered}95 \mathrm{KDa} \\ 72 \mathrm{KDa}\end{gathered}-\square=\underset{\mathrm{NSS79}}{\longleftarrow}$

IB: Flag $\underset{72 \mathrm{KDa}}{95 \mathrm{KDa}}-$

$43 \mathrm{KDa}$

IB: Actin

FIGURE 4 | NS79 decreases gcTBK1-mediated phosphorylation of gCMITA, gcIRF3, and gCIRF7. (A-C) gcTBK1 mediates the phosphorylation of gcMITA, gclRF3, and gcIRF7. GCO cells were seeded into 6-well plates overnight and transfected with the indicated plasmids ( $1.5 \mu \mathrm{g}$ each) for $24 \mathrm{~h}$. The cell lysates ( $100 \mu \mathrm{g})$ were treated with or without $\mathrm{CIP}(10 \mathrm{U})$ for $40 \mathrm{~min}$ at $37^{\circ} \mathrm{C}$. The lysates were then detected by IB with anti-Myc, anti-Flag, and anti- $\beta$-actin Abs. (D-F) gcTBK1- $\Delta \mathrm{N}$ is essential for the phosphorylation of gcMITA, gclRF3, and gclRF7. GCO cells were seeded into 6-well plates overnight and transfected with the indicated plasmids (1.5 $\mu \mathrm{g}$ each) for $24 \mathrm{~h}$. The cell lysates were subjected to IB with anti-Myc, anti-Flag, and anti- $\beta$-actin Abs. (G-I) NS79 decreases gcTBK1-mediated phosphorylation of gcMITA, gclRF3, and gCIRF7 in a dose-dependent manner. GCO cells were seeded in 6-well plates overnight and transfected with $1.5 \mu \mathrm{g}$ Flag-gcTBK1 and $1.5 \mu \mathrm{g}$ empty vector or Myc-NS79, together with $1.5 \mu \mathrm{g}$ Myc-gcMITA (G), Myc-gcIRF3 (H), or Myc-gcIRF7 (I) for $24 \mathrm{~h}$. Then the lysates were subjected to IB with anti-Myc, anti-Flag, and anti- $\beta$-actin Abs. 
A

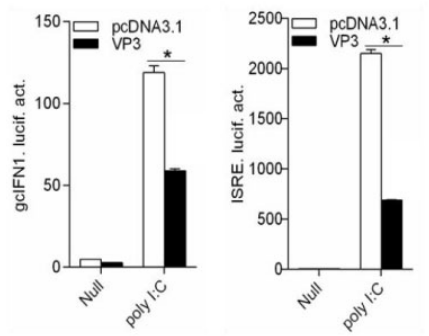

B

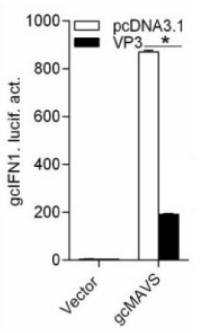

F
C

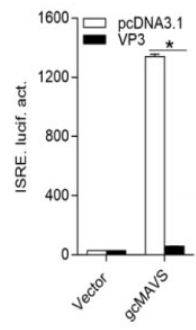

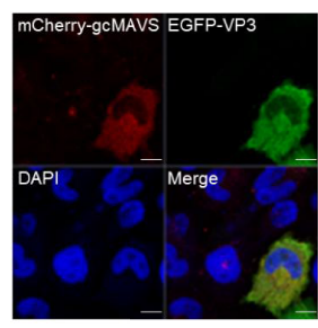

D

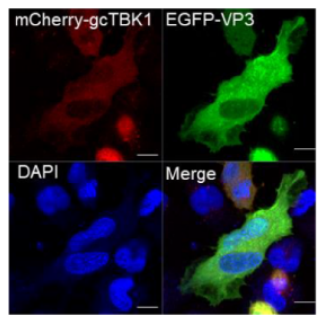

H

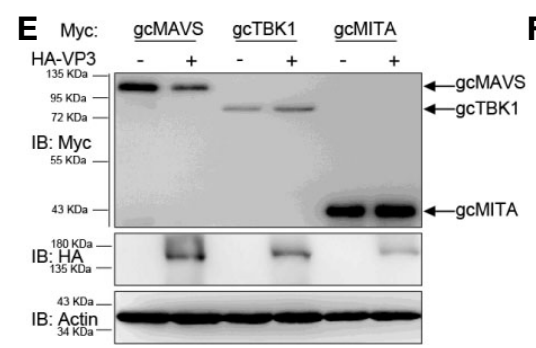

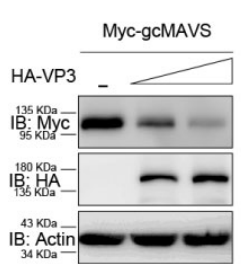

G

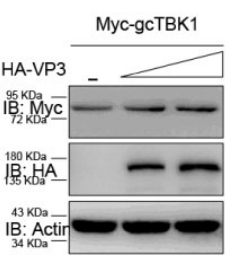

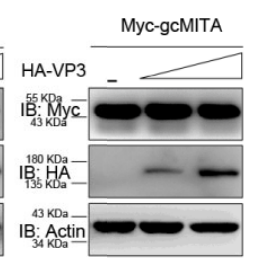
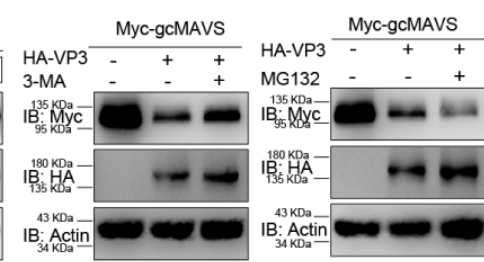

IB: Actin
I

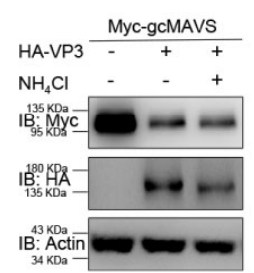

J

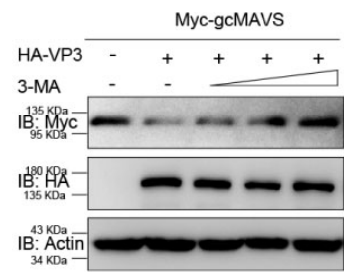

$\mathbf{K}$

K EGFP-LC3

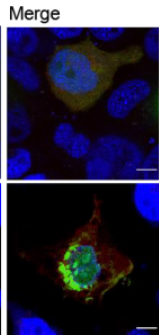

$\mathbf{L}$

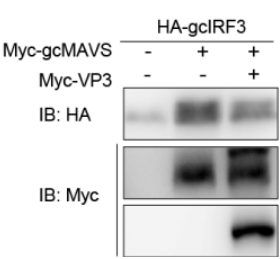

FIGURE 5 | GCRV VP3 blocks gCMAVS-mediated IFN expression and degrades gcMAVS through autophagosome pathway. (A) Overexpression of VP3 inhibits poly I:C-induced gcIFN1pro/ISRE activation. EPC cells were seeded in 24-well plates overnight and co-transfected with $0.25 \mu \mathrm{g}$ gclFN1 pro-Luc or ISRE-Luc, and 50 ng pRL-TK, plus $0.25 \mu \mathrm{g}$ empty vector or pcDNA3.1-VP3. At $24 \mathrm{~h}$ post- transfection, cells were transfected with poly l:C (1 $\mu \mathrm{g} / \mathrm{ml})$ or left untreated (null). The luciferase assay was performed $24 \mathrm{~h}$ after stimulation. (B) VP3 suppresses gcIFN1pro/ISRE activation mediated by gcMAVS. EPC cells were seeded in 24-well plates and co-transfected with gcMAVS-expressing plasmids and empty vector or pcDNA3.1-VP3, plus gcIFN1pro-Luc or ISRE-Luc at the ratio of 1:1:1. pRL-TK was used as a control. At $24 \mathrm{~h}$ post- transfection, cells were lysed for luciferase activity detection. The promoter activity is presented as relative light units normalized to Renilla luciferase activity. Data were expressed as mean $\pm \mathrm{SEM}, n=3$. Asterisks indicate significant differences from control ( $* p<0.05)$. (C, D) EPC cells were seeded on microscopy cover glass in 6-well plates and cotransfected with $2 \mu \mathrm{g}$ EGFP-VP3 and $2 \mu \mathrm{g}$ mCherry-gcMAVS (C), and mCherry-gCTBK1 (D). After $24 \mathrm{~h}$, the cells were fixed and subjected to confocal microscopy analysis. Green signals represent overexpressed VP3, red signals represent overexpressed gcMAVS or gcTBK1, and blue staining indicates the nucleus region. (original magnification, 63x oil immersion objective). Scale bar, $10 \mu \mathrm{m}$. (E) EPC cells were seeded in 6-well plates overnight and transfected with the indicated plasmids (1 $\mu \mathrm{g}$ each) for $24 \mathrm{~h}$. The cell lysates were then analyzed by IB with anti-HA, anti-Myc, and anti- $\beta$-actin Abs, respectively. (F) Overexpression of the VP3 degrades gcMAVS in a dose-dependent manner. EPC cells were seeded in 6-well plates overnight and cotransfected with $1 \mu \mathrm{g}$ Myc-gcMAVS and $1 \mu \mathrm{g}$ empty vector or HA-VP3 $(0.5$ or $1.0 \mu \mathrm{g})$ for $24 \mathrm{~h}$. Then the lysates were subjected to IB with anti-Myc, anti-HA, antiFlag, and anti- $\beta$-actin Abs. (G-I) Effects of inhibitors on VP3-mediated degradation of gcMAVS. EPC cells were seeded in 6-well plates overnight and co-transfected with $1 \mu \mathrm{g}$ Myc-gcMAVS and $1 \mu \mathrm{g}$ empty vector or HA-VP3 for $18 \mathrm{~h}$, and then treated with DMSO, MG132 (20 $\mu \mathrm{M})$, 3-MA (1 mM), or NH 4 Cl (20 mM) for $8 \mathrm{~h}$. The cell lysates were subjected to IB with anti-Myc, anti-HA, and anti- $\beta$-actin Abs. (J) 3-MA treatment recues gcMAVS degradation induced by VP3 in a dose-dependent manner. EPC cells were seeded in 6-well plates overnight and co-transfected with $1 \mu \mathrm{g}$ Myc-gcMAVS and $1 \mu \mathrm{g}$ empty vector or HA-VP3 for $18 \mathrm{~h}$, and then treated with DMSO or 3-MA $(0.5,1,2$ or $4 \mathrm{mM})$ for $8 \mathrm{~h}$. The cell lysates were subjected to IB with anti-Myc, anti-HA, and anti- $\beta$-actin Abs. (K) EPC cells seeded onto microscopy cover glass in 6-well plates were co-transfected with $1 \mu \mathrm{g}$ EGFP-LC3, $1 \mu \mathrm{g}$ mCherry-gcMAVS and pCMV-HA or HA-VP3 for $24 \mathrm{~h}$. Cells were fixed and subjected to confocal microscopy analysis. Green signals represent overexpressed LC3, red signals represent overexpressed gcMAVS, and blue staining indicates the nucleus region (original magnification, x63; oil immersion objective). Scale bar, $10 \mu \mathrm{m}$. (L) VP3 decreases gcMAVS-mediated phosphorylation of gcIRF3. HEK $293 \mathrm{~T}$ cells were seeded in 6-well plates overnight and transfected with $1 \mu \mathrm{g}$ Myc-gcMAVS and $1 \mu \mathrm{g}$ empty vector or Myc-VP3, together with $1 \mu \mathrm{g}$ HA-gclRF3 for $24 \mathrm{~h}$. The cell lysates were subjected to IB with anti-Myc, anti-HA, and anti- $\beta$-actin Abs.

VP3, but the contents of other RLR factors hardly changed (Figure 5E). In addition, the exogenous gcMAVS was further reduced with overexpressed VP3 in a dose-dependent manner, whereas no change observed in exogenous gcTBK1 and gcMITA protein levels (Figure 5F). Protein degradation is one of the main mechanisms involved in modulating protein functions in biological processes. In general, there are three systems for protein degradation: the ubiquitin-proteasome, autophagosome, and lysosomal pathways. To further elucidate the degradation system for gcMAVS, the cells were treated with the indicated inhibitors. 
The VP3-induced degradation of gcMAVS was completely blocked by the autophagosome inhibitor 3-MA, but not MG132 and $\mathrm{NH} 4 \mathrm{Cl}$, meaning that the gcMAVS degradation was mediated by VP3 via the autophagosome pathway (Figures 5G-I). In addition, gcMAVS levels were gradually rescued with increasing concentration of 3-MA, suggesting that gcMAVS degradation is mediated by VP3 via autophagosome-dependent manner (Figure 5J). To further confirm the evidence of VP3-induced autophagosome formation, confocal microcopy analysis was used to detect GFP-LC3 distribution. In control cells, GFP-LC3 was diffusely distributed, whereas the number of LC3 puncta was significantly increased in cells co-transfected with EGFP-LC3, mCherry-gcMAVS, and HA-VP3, suggesting that autophagosome formation is promoted by overexpression of VP3 (Figure 5K). The red signal from gcMAVS observed in the cytosol had no significant change after VP3 stimulation. Because gcMAVS was degraded by
VP3, we speculated that the phosphorylation of gcIRF3 might be impaired. As shown in Figure 5L, the phosphorylation of gcIRF3 mediated by gcMAVS was decreased by overexpression of VP3. Collectively, these data suggest that VP3 represses IFN induction by degrades gcMAVS in an autophagosome-dependent pathway.

\section{GCRV NS79 and VP3 Attenuate the Cellular Antiviral Response}

To ascertain whether GCRV NS79 or VP3 interferes with the cellular IFN response to facilitate virus replication, EPC cells were transfected with NS79 or VP3 and infected with SVCV. As shown in Figure 6A, a stronger CPE was observed in the NS79 and VP3 group at $48 \mathrm{~h}$ post-infection. These results were confirmed by the titers of SVCV, which significantly increased 80-fold and 300fold respectively in the NS79 and VP3-overexpressing cells compared with the control cells (Figure 6B). In addition, qPCR
A

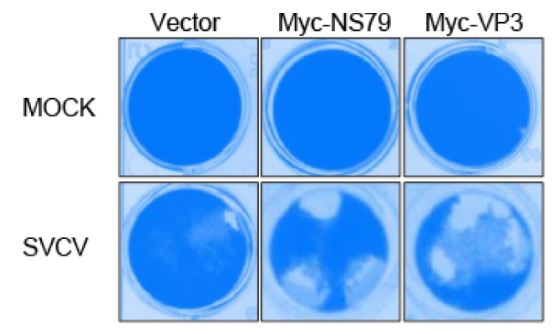

C

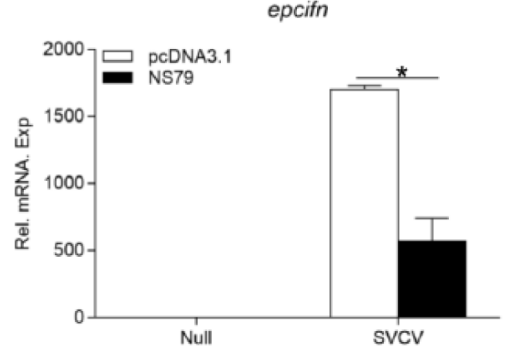

D

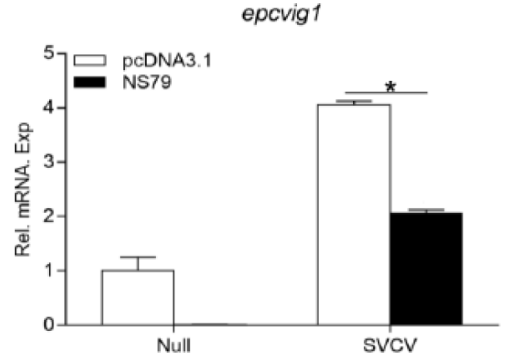

B
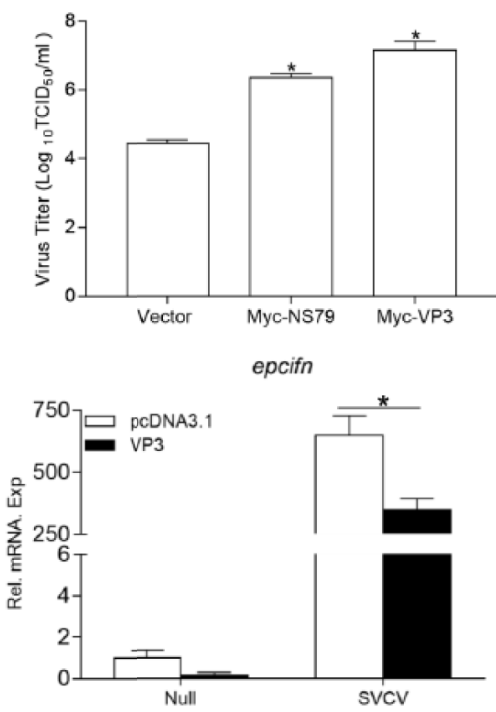

epcrig1

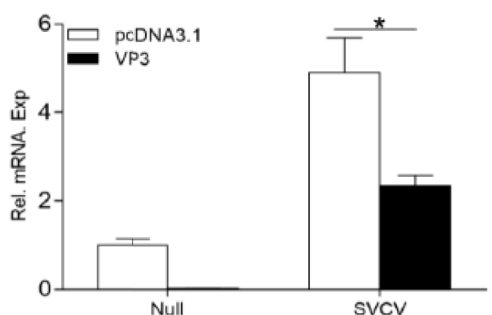

FIGURE 6 | Overexpression of GCRV NS79 or VP3 dampens the cellular IFN responses. (A, B) Increase of virus replication by overexpression of NS79 or VP3. EPC cells were seeded in 24-well plates overnight and transfected with indicated plasmids for $24 \mathrm{~h}$, then the cells were infected with SVCV (MOI = 0.001 ) for $48 \mathrm{~h}$. (A) Then, the cells were fixed with 4\% PFA and stained with 1\% crystal violet. (B) Culture supernatants from the cells infected with SVCV were collected, and the viral titer was measured according to the method of Reed and Muench. (C, D) overexpression of NS79 or VP3 inhibits SVCV-induced up-regulation of epcifn (C) and epcvig1 (D). EPC cells seeded in 6-well plates overnight were transfected with $2 \mu \mathrm{g}$ empty vector or pcDNA3.1-NS79, or pcDNA3.1-VP3, at 24 h post-transfection, the cells were infected with SVCV $(\mathrm{MOI}=1)$ for $24 \mathrm{~h}$. The total RNAs were extracted to examine the mRNA levels of cellular epcifn and epcvig1. The relative transcriptional levels were normalized to the transcriptional level of the $\beta$-actin gene and were represented as fold induction relative to the transcriptional level in the control cells, which was set to 1. Data were expressed as mean \pm SEM, $n=3$. Asterisks indicate significant differences from control values $\left({ }^{\star} p<0.05\right)$. 
analysis demonstrated that the overexpression of NS79 or VP3 blocked the SVCV-induced expression of ifn and vig1 (Figures 6C, D). These data indicate that GCRV NS79 and VP3 suppress the cellular IFN response and facilitate SVCV proliferation.

\section{DISCUSSION}

In a water living environment, aquatic viruses can spread more easily and cause higher mortality than land-based viruses. The possible reasons likely depending on many factors, including host species (lower vertebrates or higher vertebrates), host life history, environment, degree of anthropogenic manipulation, and so on (47). During that long period of evolutionary time, viruses have had to adapt to markedly different hosts. In previous studies, our lab has reported that GCRV VP41 protein (encoded by the S8 segment) as well as SVCV $\mathrm{N}$ and $\mathrm{P}$ proteins antagonize fish RLR factors to reduce host IFN production (43-45), highlighting the evasion mechanisms used by aquatic viruses. To date, precise information regarding both the fish IFN response to viruses and the pathogenesis of aquatic viruses is rare; thus, the immune evasion mechanisms of aquatic viruses targeting the host IFN system are unclear. Here, we report that all GCRV viral proteins can interact with host RLR factors, specifically inhibiting the MAVS-mediated production of IFNs. Furthermore, we found that NS79 reduces gcMITA phosphorylation by acting as a decoy substrate of gcTBK1 while VP3 degrades gcMAVS in an autophagosome-dependent manner, ultimately inhibiting IFN production and facilitating virus replication. Though Co-IP data were successfully obtained in HEK 293T cells, the difference between mammalian and fish cells should be further explored. In conclusion, these findings enhance the understanding of the immune evasion mechanisms of GCRV.

It is obviously rare for all proteins in one virus to interfere with the TBK1 kinase, hence we presume the observed phenomenon has two causes: (1) TBK1 is crucial for fish IFN production. More type I IFN members are found in fish than in mammals (which possess only IFN $\alpha$ and IFN $\beta$ ), and the regulation patterns are more complicated. TBK1 is the upstream kinase of IRF3, IRF7, and even IRF6 (positive regulator of fish IFNs), displaying a powerful capacity to activate IFNs (48); therefore, targeting TBK1 is more efficient for a virus than targeting each IRF. (2) TBK1 has multiple functions in hosts. Besides activating IFN transcription, TBK1 participates in several host life processes such as cellular transformation, autophagy, antibacterial response, and oncogenesis $(28,49)$. For example, autophagy is a conserved process in eukaryotic cells and plays a crucial role in the eukaryotic defense against pathogens. TBK1 interacts with and phosphorylates optineurin (OPTN, a key component of pathogen-induced autophagy), leading to the elimination of pathogens by xenophagy (50). For viral infection, viruses also need to control host resources for replication and proliferation in addition to combating the host IFN response. In addition, TBK1 is downstream cascade of MAVS, though all GCRV proteins interacts with TBK1, therefore, the IFN induction mediated by MAVS are inhibited but not by TBK1. On the other hand, if TBK1 function is antagonized by GCRV proteins, overexpression of TBK1 could rescue such inhibition resulting the almost normal expression of IFN. Thus, choosing TBK1 as the target is a highly effective way for GCRV to proliferate in host cells. The cell physiology mediated by GCRV-regulated TBK1 should be elucidated with further studies on the biological function of fish TBK1.

TBK1 is a pivotal protein kinase that is utilized by viruses (51). In the viral lifecycle, the phosphorylation of viral proteins has been identified as indispensable, and several studies have suggested that viral proteins are not active until phosphorylated by cellular kinase. The phosphorylation of NS1 protein from Periplaneta fuliginosa densovirus (PfDNV) triggers the activation of viral genome replication and transcription (52). IE63 from vesicular stomatitis virus (VSV) is phosphorylated by host cellular cyclin-dependent kinase (CDK) 1 and CDK2, then translocates from the nucleus to the cytoplasm (53). Human immunodeficiency virus 1 (HIV-1) Gag and $\mathrm{Vpr}$ proteins improve their assembly into viral particles after phosphorylation by host atypical protein kinase C (aPKC) (54). The phosphorylation of viral proteins has also been observed in aquatic viruses. For example, SVCV P protein can be phosphorylated by TBK1, which leads to the decline of IRF3 phosphorylation and IFN production (44). However, the function of phosphorylated $\mathrm{P}$ protein in SVCV proliferation is still unknown. In this study, the NS79 protein encoded by the GCRV S4 segment was also phosphorylated by TBK1. As S4 encodes a non-structural protein and is possibly involved in the formation of viral inclusion bodies, with the protein encoded by GCRV S9, the function of phosphorylated NS79 might be indispensable for viral assembly.

Conversely, the physical binding of TBK1 with viral proteins might inhibit the host's antiviral response. Since TBK1 is considered to have a pivotal antiviral role in phosphorylating IRF3 to activate IFN transcription, the significant disruption of the signaling transduction of TBK1 by viruses will blunt the host IFN production (25). Meanwhile, the viral protein(s) that interact with host TBK1 may also be crucial for the viral life cycle, and such neutralization will disrupt the normal transcription, translation, and proliferation of viruses. For example, the borna disease virus (BDV) $\mathrm{P}$ protein associates with $\mathrm{TBK} 1$ and inhibits its kinase activity to promote viral evasion (55). The rhabdovirus $\mathrm{P}$ protein interacts with the $\mathrm{L}$ protein binding with the viral template in the transcription process, which facilitates the $\mathrm{N}$ protein staying in a soluble, encapsidation-competent form that is associated with viral RNA to form the nucleocapsid during viral assembly $(56,57)$. The $\mathrm{P}$ protein amount is reduced after reacting with the host TBK1; a lower concentration of the $\mathrm{P}$ protein should reduce the normal viral transcription level. The outcome of the combat between virus and host might be determined by the amount and role of the proteins that participate on both sides, as well as the reaction efficiency; however, the exact mechanisms need to be further clarified. The current study identified a novel phenomenon of aquatic virus GCRV countering the host IFN response. In the most common and highest mortality genotype of GCRV, GCRV II, all the viral proteins encoded by the segments reduce the host IFN transcription by interacting with TBK1. We hope our findings provide a base for further study of GCRV evasion mechanisms related to TBK1.

The critical role of MAVS in the production of IFN and other proinflammatory cytokines predisposes it to being a target of 
many viruses (58). In long-term coexistence of virus and host, viruses have evolved various strategies to suppress MAVSmediated signaling. One of the most common mechanisms is the cleavage of MAVS, resulting in the dislocation of MAVS from the mitochondria, thus preventing IFN induction. For instance, the $\mathrm{HCV}$ protease NS3/4A and Enterovirus 71 Protease 2Apro cleave MAVS to block signaling transduction $(59,60)$. Besides the cleavage of MAVS, several viruses choose to degrade MAVS. For example, hepatitis B virus X protein (HBX) interacts with MAVS, inducing the degradation of MAVS through Lys136 ubiquitin in MAVS protein, thus inhibiting IFN expression (61). Our finding that GCRV VP3 interacts with and degrades MAVS in an autophagosome-dependent manner provides new insight into how virus-derived proteins and MAVS can interact.

Overall, we showed that GCRV attenuates host immune signaling mediated by two potent antiviral adapter molecules, MAVS and TBK1. This is achieved using two distinct methods to reduce MAVS and TBK1 signaling, namely VP3 triggering the degradation of MAVS and NS79 serving as a substrate of TBK1 to reduce IRF3 phosphorylation. Our findings therefore revealed new mechanisms of GCRV-mediated evasion of the host's innate immunity.

\section{AUTHOR'S NOTE}

This manuscript has been released as a pre-print at bioRxiv.

\section{DATA AVAILABILITY STATEMENT}

The original contributions presented in the study are included in the article/Supplementary Materials. Further inquiries can be directed to the corresponding authors.

\section{REFERENCES}

1. Kawai T, Akira S. Innate immune recognition of viral infection. Nat Immunol (2006) 7(2):131-7. doi: 10.1038/ni1303

2. Zou J, Secombes CJ. Teleost fish interferons and their role in immunity. Dev Comp Immunol (2011) 35(12):1376-87. doi: 10.1016/j.dci.2011.07.001

3. Gurtler C, Bowie AG. Innate immune detection of microbial nucleic acids. Trends Microbiol (2013) 21(8):413-20. doi: 10.1016/j.tim.2013. 04.004

4. Langevin C, Aleksejeva E, Passoni G, Palha N, Levraud JP, Boudinot P. The antiviral innate immune response in fish: evolution and conservation of the IFN system. J Mol Biol (2013) 425(24):4904-20. doi: 10.1016/j.jmb.2013. 09.033

5. Zou J, Tafalla C, Truckle J, Secombes CJ. Identification of a second group of type I IFNs in fish sheds light on IFN evolution in vertebrates. J Immunol (2007) 179(6):3859-71. doi: 10.4049/jimmunol.179.6.3859

6. Sun B, Robertsen B, Wang Z, Liu B. Identification of an Atlantic salmon IFN multigene cluster encoding three IFN subtypes with very different expression properties. Dev Comp Immunol (2009) 33(4):547-58. doi: 10.1016/ j.dci.2008.10.001

7. Akira $S$, Uematsu S, Takeuchi O. Pathogen recognition and innate immunity. Cell (2006) 124(4):783-801. doi: 10.1016/j.cell.2006.02.015

8. Loo YM, Gale M. Immune Signaling by RIG-I-like Receptors. Immunity (2011) 34(5):680-92. doi: 10.1016/j.immuni.2011.05.003

\section{AUTHOR CONTRIBUTIONS}

$\mathrm{Y}-\mathrm{AZ}$ and SL conceived and designed the experiments. SL, L-FL, Z-CL, CZ, X-YZ, YZ, J-YJ, and D-DC performed the experiments and analyzed the data. SL, L-FL, and Z-CL wrote the manuscript. All authors contributed to the article and approved the submitted version.

\section{FUNDING}

This work was supported by National Key Research and Development Program of China (2018YFD0900504) provided to Shun Li. National Natural Science Foundation of China (31725026) and the Science Fund for Creative Research Groups of the Natural Science Foundation of Hubei Province of China (2018CFA011) provided to Y-AZ. Youth Innovation Promotion Association provided to SL, and National Natural Science Foundation of China (31802338) provided to L-FL.

\section{ACKNOWLEDGMENTS}

We thank Dr. Fang Zhou (Institute of Hydrobiology, Chinese Academy of Sciences) for assistance with confocal microscopy analysis.

\section{SUPPLEMENTARY MATERIAL}

The Supplementary Material for this article can be found online at: https://www.frontiersin.org/articles/10.3389/fimmu.2020. 545302/full\#supplementary-material

9. Kawai T, Takahashi K, Sato S, Coban C, Kumar H, Kato H, et al. IPS-1, an adaptor triggering RIG-I- and Mda5-mediated type I interferon induction. Nat Immunol (2005) 6(10):981-8. doi: 10.1038/ni1243

10. Meylan E, Curran J, Hofmann K, Moradpour D, Binder M, Bartenschlager R, et al. Cardif is an adaptor protein in the RIG-I antiviral pathway and is targeted by hepatitis C virus. Nature (2005) 437(7062):1167-72. doi: 10.1038/ nature 04193

11. Seth RB, Sun L, Ea CK, Chen ZJ. Identification and characterization of MAVS, a mitochondrial antiviral signaling protein that activates NFkappaB and IRF 3. Cell (2005) 122(5):669-82. doi: 10.1016/j.cell. 2005.08.012

12. Xu LG, Wang YY, Han KJ, Li LY, Zhai Z, Shu HB. VISA is an adapter protein required for virus-triggered IFN-beta signaling. Mol Cell (2005) 19(6):727-40. doi: 10.1016/j.molcel.2005.08.014

13. Zhong B, Yang Y, Li S, Wang YY, Li Y, Diao F, et al. The adaptor protein MITA links virus-sensing receptors to IRF3 transcription factor activation. Immunity (2008) 29(4):538-50. doi: 10.1016/j.immuni.2008.09.003

14. Ishikawa H, Barber GN. STING is an endoplasmic reticulum adaptor that facilitates innate immune signalling. Nature (2008) 455(7213):674-8. doi: $10.1038 /$ nature 07317

15. Jin L, Waterman PM, Jonscher KR, Short CM, Reisdorph NA, Cambier JC. MPYS, a novel membrane tetraspanner, is associated with major histocompatibility complex class II and mediates transduction of apoptotic signals. Mol Cell Biol (2008) 28(16):5014-26. doi: 10.1128/Mcb.00640-08 
16. Sun WX, Li Y, Chen L, Chen HH, You FP, Zhou X, et al. ERIS, an endoplasmic reticulum IFN stimulator, activates innate immune signaling through dimerization. Proc Natl Acad Sci USA (2009) 106(21):8653-8. doi: 10.1073/ pnas.0900850106

17. Fitzgerald KA, McWhirter SM, Faia KL, Rowe DC, Latz E, Golenbock DT, et al. IKKepsilon and TBK1 are essential components of the IRF3 signaling pathway. Nat Immunol (2003) 4(5):491-6. doi: 10.1038/ni921

18. Schafer SL, Lin R, Moore PA, Hiscott J, Pitha PM. Regulation of type I interferon gene expression by interferon regulatory factor-3. J Biol Chem (1998) 273(5):2714-20. doi: 10.1074/jbc.273.5.2714

19. Genin P, Lin R, Hiscott J, Civas A. Differential regulation of human interferon A gene expression by interferon regulatory factors 3 and 7. Mol Cell Biol (2009) 29(12):3435-50. doi: 10.1128/MCB.01805-08

20. Sun Q, Sun L, Liu HH, Chen X, Seth RB, Forman J, et al. The specific and essential role of MAVS in antiviral innate immune responses. Immunity (2006) 24(5):633-42. doi: 10.1016/j.immuni.2006.04.004

21. Moore CB, Ting JP. Regulation of mitochondrial antiviral signaling pathways. Immunity (2008) 28(6):735-9. doi: 10.1016/j.immuni.2008.05.005

22. Huang YF, Liu H, Li SL, Tang YJ, Wei B, Yu HS, et al. MAVS-MKK7-JNK2 Defines a Novel Apoptotic Signaling Pathway during Viral Infection. PLoS Pathog (2014) 10(3):e1004020. doi: 10.1371/journal.ppat.1004020.

23. Lauksund S, Svingerud T, Bergan V, Robertsen B. Atlantic salmon IPS-1 mediates induction of IFNal and activation of NF-kappaB and localizes to mitochondria. Dev Comp Immunol (2009) 33(11):1196-204. doi: 10.1016/ j.dci.2009.06.012

24. Biacchesi S, LeBerre M, Lamoureux A, Louise Y, Lauret E, Boudinot P, et al. Mitochondrial antiviral signaling protein plays a major role in induction of the fish innate immune response against RNA and DNA viruses. J Virol (2009) 83(16):7815-27. doi: 10.1128/JVI.00404-09

25. Zhao W. Negative regulation of TBK1-mediated antiviral immunity. FEBS Lett (2013) 587(6):542-8. doi: 10.1016/j.febslet.2013.01.052

26. Hu YW, Zhang J, Wu XM, Cao L, Nie P, Chang MX. TANK-Binding Kinase 1 (TBK1) Isoforms Negatively Regulate Type I Interferon Induction by Inhibiting TBK1-IRF3 Interaction and IRF3 Phosphorylation. Front Immunol (2018) 9:84. doi: 10.3389/Fimmu.2018.00084

27. Pilli M, Arko-Mensah J, Ponpuak M, Roberts E, Master S, Mandell MA, et al. TBK-1 promotes autophagy-mediated antimicrobial defense by controlling autophagosome maturation. Immunity (2012) 37(2):223-34. doi: 10.1016/ j.immuni.2012.04.015

28. Weidberg H, Elazar Z. TBK1 mediates crosstalk between the innate immune response and autophagy. Sci Signal (2011) 4(187):pe39. doi: 10.1126/ scisignal.2002355

29. Feng X, Su J, Yang C, Yan N, Rao Y, Chen X. Molecular characterizations of grass carp (Ctenopharyngodon idella) TBK1 gene and its roles in regulating IFN-I pathway. Dev Comp Immunol (2014) 45(2):278-90. doi: 10.1016/ j.dci.2014.03.018

30. Zhang L, Chen WQ, Hu YW, Wu XM, Nie P, Chang MX. TBK1-like transcript negatively regulates the production of IFN and IFN-stimulated genes through RLRs-MAVS-TBK1 pathway. Fish Shellfish Immunol (2016) 54:135-43. doi: 10.1016/j.fsi.2016.04.002

31. Sun F, Zhang YB, Liu TK, Shi J, Wang B, Gui JF. Fish MITA serves as a mediator for distinct fish IFN gene activation dependent on IRF3 or IRF7. J Immunol (2011) 187(5):2531-9. doi: 10.4049/jimmunol.1100642

32. Sun Y, Zheng H, Yu S, Ding Y, Wu W, Mao X, et al. Newcastle Disease Virus V Protein Degrades Mitochondrial Antiviral Signaling Protein To Inhibit Host Type I Interferon Production via E3 Ubiquitin Ligase RNF5. J Virol (2019) 93(18):e00322-19. doi: 10.1128/JVI.00322-19

33. Mukherjee A, Morosky SA, Delorme-Axford E, Dybdahl-Sissoko N, Oberste MS, Wang T, et al. The coxsackievirus B 3C protease cleaves MAVS and TRIF to attenuate host type I interferon and apoptotic signaling. PLoS Pathog (2011) 7(3):e1001311. doi: 10.1371/journal.ppat. 1001311

34. Dong JM, Xu SG, Wang J, Luo R, Wang D, Xiao SB, et al. Porcine reproductive and respiratory syndrome virus $3 \mathrm{C}$ protease cleaves the mitochondrial antiviral signalling complex to antagonize IFN-beta expression. J Gen Virol (2015) 96:3049-58. doi: 10.1099/jgv.0.000257

35. Otsuka M, Kato N, Moriyama M, Taniguchi H, Wang Y, Dharel N, et al. Interaction between the HCV NS3 protein and the host TBK1 protein leads to inhibition of cellular antiviral responses. Hepatology (2005) 41(5):1004-12. doi: 10.1002/hep.20666

36. Wang D, Fang LR, Li P, Sun L, Fan JX, Zhang QY, et al. The Leader Proteinase of Foot-and-Mouth Disease Virus Negatively Regulates the Type I Interferon Pathway by Acting as a Viral Deubiquitinase. J Virol (2011) 85(8):3758-66. doi: 10.1128/Jvi.02589-10

37. Rangel AAC, Rockemann DD, Hetrick FM, Samal SK. Identification of grass carp haemorrhage virus as a new genogroup of aquareovirus. J Gen Virol (1999) 80:2399-402. doi: 10.1099/0022-1317-80-9-2399

38. Wang Q, Zeng WW, Liu C, Zhang C, Wang YY, Shi CB, et al. Complete Genome Sequence of a Reovirus Isolated from Grass Carp, Indicating Different Genotypes of GCRV in China. J Virol (2012) 86(22):12466-. doi: 10.1128/Jvi.02333-12

39. Attoui H, Fang Q, Jaafar FM, Cantaloube JF, Biagini P, de Micco P, et al. Common evolutionary origin of aquareoviruses and orthoreoviruses revealed by genome characterization of Golden shiner reovirus, Grass carp reovirus, Striped bass reovirus and golden ide reovirus (genus Aquareovirus, family Reoviridae). J Gen Virol (2002) 83:1941-51. doi: 10.1099/0022-1317-83-8-1941

40. Cheng LP, Fang Q, Shah S, Atanasov IC, Zhou ZH. Subnanometer-resolution structures of the grass carp reovirus core and virion. J Mol Biol (2008) 382 (1):213-22. doi: 10.1016/j.jmb.2008.06.075

41. Peng L, Yang C, Su J. Protective roles of grass carp Ctenopharyngodon idella Mx isoforms against grass carp reovirus. PLoS One (2012) 7(12):e52142. doi: 10.1371/journal.pone.0052142

42. Wang B, Zhang YB, Liu TK, Shi J, Sun F, Gui JF. Fish viperin exerts a conserved antiviral function through RLR-triggered IFN signaling pathway. Dev Comp Immunol (2014) 47(1):140-9. doi: 10.1016/j.dci.2014.07.006

43. Lu LF, Li S, Wang ZX, Du SQ, Chen DD, Nie P, et al. Grass Carp Reovirus VP41 Targets Fish MITA To Abrogate the Interferon Response. J Virol (2017) 91(14):e00390-17. doi: 10.1128/JVI.00390-17

44. Li S, Lu LF, Wang ZX, Lu XB, Chen DD, Nie P, et al. The P Protein of Spring Viremia of Carp Virus Negatively Regulates the Fish Interferon Response by Inhibiting the Kinase Activity of TANK-Binding Kinase 1. J Virol (2016) 90 (23):10728-37. doi: 10.1128/JVI.01381-16

45. Lu LF, Li S, Lu XB, LaPatra SE, Zhang N, Zhang XJ, et al. Spring Viremia of Carp Virus N Protein Suppresses Fish IFNphil Production by Targeting the Mitochondrial Antiviral Signaling Protein. J Immunol (2016) 196(9):3744-53. doi: 10.4049/jimmunol.1502038

46. Pei C, Ke F, Chen ZY, Zhang QY. Complete genome sequence and comparative analysis of grass carp reovirus strain 109 (GCReV-109) with other grass carp reovirus strains reveals no significant correlation with regional distribution. Arch Virol (2014) 159(9):2435-40. doi: 10.1007/s00705-014-2007-5

47. Purcell MK, Laing KJ, Winton JR. Immunity to Fish Rhabdoviruses. VirusesBasel (2012) 4(1):140-66. doi: 10.3390/v4010140

48. Li S, Lu LF, Wang ZX, Chen DD, Zhang YA. Fish IRF6 is a positive regulator of IFN expression and involved in both of the MyD88 and TBK1 pathways. Fish Shellfish Immunol (2016) 57:262-8. doi: 10.1016/j.fsi.2016.08.059

49. Thurston TL, Ryzhakov G, Bloor S, von Muhlinen N, Randow F. The TBK1 adaptor and autophagy receptor NDP52 restricts the proliferation of ubiquitin-coated bacteria. Nat Immunol (2009) 10(11):1215-21. doi: 10.1038/ ni. 1800

50. Wild P, Farhan H, McEwan DG, Wagner S, Rogov VV, Brady NR, et al. Phosphorylation of the autophagy receptor optineurin restricts Salmonella growth. Science (2011) 333(6039):228-33. doi: 10.1126/science.1205405

51. Taylor KE, Mossman KL. Recent advances in understanding viral evasion of type I interferon. Immunology (2013) 138(3):190-7. doi: 10.1111/imm.12038

52. Han $\mathrm{Y}$, Wang Q, Qiu $\mathrm{Y}, \mathrm{Wu} \mathrm{W}, \mathrm{He} \mathrm{H}$, Zhang J, et al. Periplaneta fuliginosa densovirus nonstructural protein NS1 contains an endonuclease activity that is regulated by its phosphorylation. Virology (2013) 437(1):1-11. doi: 10.1016/ j.virol.2012.12.006

53. Habran L, Bontems S, Di Valentin E, Sadzot-Delvaux C, Piette J. Varicellazoster virus IE63 protein phosphorylation by roscovitine-sensitive cyclindependent kinases modulates its cellular localization and activity. J Biol Chem (2005) 280(32):29135-43. doi: 10.1074/jbc.M503312200

54. Kudoh A, Takahama S, Sawasaki T, Ode H, Yokoyama M, Okayama A, et al. The phosphorylation of HIV-1 Gag by atypical protein kinase C facilitates viral infectivity by promoting $\mathrm{Vpr}$ incorporation into virions. Retrovirology (2014) 11:9. doi: 10.1186/1742-4690-11-9 
55. Unterstab G, Ludwig S, Anton A, Planz O, Dauber B, Krappmann D, et al. Viral targeting of the interferon-\{beta\}-inducing Traf family member-associated NF- $\{$ kappa $\}$ B activator (TANK)-binding kinase-1. Proc Natl Acad Sci USA (2005) 102(38):13640-5. doi: 10.1073/pnas. 0502883102

56. Green TJ, Zhang X, Wertz GW, Luo M. Structure of the vesicular stomatitis virus nucleoprotein-RNA complex. Science (2006) 313(5785):357-60. doi: 10.1126/science.1126953

57. Rahmeh AA, Morin B, Schenk AD, Liang B, Heinrich BS, Brusic V, et al. Critical phosphoprotein elements that regulate polymerase architecture and function in vesicular stomatitis virus. Proc Natl Acad Sci USA (2012) 109 (36):14628-33. doi: 10.1073/pnas.1209147109

58. Bhoj VG, Sun QM, Bhoj EJ, Somers C, Chen X, Torres JP, et al. MAVS and MyD88 are essential for innate immunity but not cytotoxic T lymphocyte response against respiratory syncytial virus. Proc Natl Acad Sci USA (2008) 105(37):14046-51. doi: 10.1073/pnas.0804717105

59. Li XD, Sun L, Seth RB, Pineda G, Chen ZJ. Hepatitis C virus protease NS3/4A cleaves mitochondrial antiviral signaling protein off the mitochondria to evade innate immunity. Proc Natl Acad Sci USA (2005) 102(49):17717-22. doi: $10.1073 /$ pnas. 0508531102
60. Wang B, Xi XY, Lei XB, Zhang XY, Cui S, Wang JW, et al. Enterovirus 71 Protease $2 \mathrm{~A}$ (pro) Targets MAVS to Inhibit Anti-Viral Type I Interferon Responses. PLoS Pathog (2013) 9(3):e1003231. doi: 10.1371/journal.ppat. 1003231

61. Wei C, Ni C, Song T, Liu Y, Yang X, Zheng Z, et al. The hepatitis B virus X protein disrupts innate immunity by downregulating mitochondrial antiviral signaling protein. J Immunol (2010) 185(2):1158-68. doi: 10.4049/ jimmunol.0903874

Conflict of Interest: The authors declare that the research was conducted in the absence of any commercial or financial relationships that could be construed as a potential conflict of interest.

Copyright $\odot 2020 \mathrm{Lu}, \mathrm{Li}$, Zhang, Zhou, Zhou, Jiang, Chen, Li and Zhang. This is an open-access article distributed under the terms of the Creative Commons Attribution License (CC BY). The use, distribution or reproduction in other forums is permitted, provided the original author(s) and the copyright owner(s) are credited and that the original publication in this journal is cited, in accordance with accepted academic practice. No use, distribution or reproduction is permitted which does not comply with these terms. 